Supplement of

\title{
Global atmospheric carbon monoxide budget 2000-2017 inferred from multi-species atmospheric inversions
}

Bo Zheng et al.

Correspondence to: Bo Zheng (bo.zheng@ @1sce.ipsl.fr)

The copyright of individual parts of the supplement might differ from the CC BY 4.0 License. 
Table S1. Error settings in the covariance matrices $B$ and $R$.

\begin{tabular}{|c|c|c|}
\hline Model variable & Error settings & Main reference \\
\hline \multicolumn{3}{|l|}{ Covariance matrix B } \\
\hline Gridded CO emissions & $100 \%$ of the largest emission flux in the grid cell within the year & Yin et al. (2015) \\
\hline Gridded $\mathrm{CH}_{4}$ emissions & $70 \%$ of the largest emission flux among the nine grid cells around & $100 \%$ in Yin et al. (2015) \\
\hline Gridded MCF emissions & $10 \%$ & Yin et al. (2015) \\
\hline Gridded scaling factors to & $200 \%$ & $100 \%$ in Yin et al. (2015) \\
\hline \multicolumn{3}{|l|}{ HCHO produced by NMVOCs } \\
\hline \multicolumn{3}{|l|}{$\begin{array}{l}\text { model initial concentrations of } \\
\mathrm{CO}, \mathrm{CH}_{4}, \mathrm{MCF} \text {, and } \mathrm{HCHO}\end{array}$} \\
\hline Scaling factors to the $\mathrm{OH}$ fields & $5 \%$ & $10 \%$ in Yin et al. (2015) \\
\hline \multicolumn{3}{|l|}{ Covariance matrix $\mathbf{R}$} \\
\hline CO total column & $\begin{array}{l}\text { Instrument errors are from the MOPITT satellite. The forward model and } \\
\text { representation errors are estimated as } 30 \% \text { of the measured data. }\end{array}$ & Yin et al. (2015) \\
\hline HCHO total column & $\begin{array}{l}\text { Instrument errors are from the OMI satellite. The forward model and } \\
\text { representation errors are estimated as } 30 \% \text { of the measured data. }\end{array}$ & Yin et al. (2016) \\
\hline $\mathrm{XCH}_{4}$ & $\begin{array}{l}\text { Instrument errors are from the GOSAT satellite. The forward model and } \\
\text { representation errors are estimated as } 10 \% \text { of the measured data. }\end{array}$ & Assumed \\
\hline MCF concentration & $\begin{array}{l}\text { Instrument errors are from the WDCGG dataset. The forward model and } \\
\text { representation errors are estimated as the standard deviation of de- } \\
\text { trended and de-seasonalized time series as Yin et al. (2015). }\end{array}$ & Yin et al. (2015) \\
\hline
\end{tabular}


Table S2. The WDCGG sites used for evaluating modelled surface CO concentrations.

\begin{tabular}{|c|c|c|c|c|c|c|}
\hline Site & Contributor & $\begin{array}{l}\text { Measurement } \\
\text { method }\end{array}$ & $\begin{array}{c}\text { Latitude } \\
\left({ }^{\circ}\right)\end{array}$ & $\underset{\left({ }^{\circ}\right)}{\text { Longitude }}$ & $\begin{array}{c}\text { Measurement } \\
\text { altitude (m) }\end{array}$ & Data period \\
\hline $\mathrm{CGO}$ & $\begin{array}{l}\text { AGAGE, CSIRO, } \\
\text { NOAA }\end{array}$ & surface-flask, surface-insitu & -40.68 & 144.69 & 164 & 2000-2017 \\
\hline MHD & AGAGE, NOAA & surface-flask, surface-insitu & 53.33 & -9.90 & 5 & 2000-2017 \\
\hline KVV & ARSO & surface-insitu & 46.30 & 14.53 & 1750 & 2005-2017 \\
\hline KOS & CHMI & surface-insitu & 49.58 & 15.08 & 535 & 2000-2017 except 2007 \\
\hline CFA & CSIRO & surface-flask & -19.28 & 147.06 & 5 & $2000-2017$ \\
\hline CRI & CSIRO & surface-flask & 15.08 & 73.83 & 66 & 2000-2002, 2009-2013 \\
\hline CYA & CSIRO & surface-flask & -66.28 & 110.52 & 55 & $2000-2017$ \\
\hline ESP & CSIRO & surface-flask & 49.38 & -126.54 & 47 & 2000-2002, 2009-2017 \\
\hline GPA & CSIRO & surface-flask & -12.25 & 131.05 & 37 & $2010-2015$ \\
\hline MAA & CSIRO & surface-flask & -67.60 & 62.87 & 42 & $2000-2017$ \\
\hline MQA & CSIRO & surface-flask & -54.50 & 158.94 & 13 & 2000-2017 \\
\hline SIS & CSIRO & surface-flask & 60.13 & -1.18 & 33 & $2000-2003$ \\
\hline ALT & CSIRO, NOAA & surface-flask & 82.50 & -62.34 & 203 & $2000-2017$ \\
\hline MLO & CSIRO, NOAA & surface-flask & 19.54 & -155.58 & 3435 & 2000-2017 \\
\hline SPO & CSIRO, NOAA & surface-flask & -90.00 & -24.80 & 2847 & $2000-2017$ \\
\hline TLL & DMC & surface-insitu & -30.17 & -70.80 & 2159 & 2013-2015 \\
\hline GAT & DWD & tower-insitu & 53.07 & 11.44 & 226 & 2017 \\
\hline $\mathrm{CDL}$ & ECCC & surface-insitu & 53.99 & -105.12 & 630 & $2002-2007$ \\
\hline $\mathrm{CHL}$ & ECCC & surface-insitu & 58.74 & -93.82 & 89 & 2013-2017 \\
\hline CHM & ECCC & surface-insitu & 49.69 & -74.34 & 423 & 2007-2010 \\
\hline EGB & ECCC & surface-insitu & 44.23 & -79.78 & 255 & 2005-2017 \\
\hline ETL & ECCC & surface-insitu & 54.35 & -104.99 & 598 & 2005-2017 \\
\hline FSD & ECCC & surface-insitu & 49.84 & -81.52 & 250 & 2002-2017 \\
\hline WSA & ECCC & surface-insitu & 43.93 & -60.01 & 30 & $2003-2013$ \\
\hline JFJ & Empa & surface-insitu & 46.55 & 7.99 & 3585 & $2000-2017$ \\
\hline PAY & Empa & surface-insitu & 46.81 & 6.94 & 495 & $2000-2017$ \\
\hline RIG & Empa & surface-insitu & 47.07 & 8.46 & 1031 & 2000-2017 \\
\hline CUR & IIA & surface-insitu & 39.32 & 16.42 & 1801 & 2014-2017 \\
\hline $\mathrm{ABP}$ & INPE, NOAA & surface-flask & -12.77 & -38.17 & 11 & $2006-2010$ \\
\hline BEO & INRNE & surface-insitu & 42.18 & 23.59 & 2931 & 2006-2012 \\
\hline CGR & ISAC & surface-insitu & 37.67 & 12.65 & 9 & 2015-2017 \\
\hline $\mathrm{ECO}$ & ISAC & surface-insitu & 40.34 & 18.12 & 43 & 2015,2016 \\
\hline LMT & ISAC & surface-insitu & 38.88 & 16.23 & 14 & 2015,2016 \\
\hline MNM & JMA & surface-insitu & 24.29 & 153.98 & 27 & $\begin{array}{c}2000-2017 \text { except } 2004 \\
\text { and } 2005\end{array}$ \\
\hline RYO & JMA & surface-insitu & 39.03 & 141.82 & 280 & $2000-2017$ \\
\hline YON & JMA & surface-insitu & 24.47 & 123.01 & 50 & $2000-2017$ \\
\hline PDM & LA & surface-insitu & 42.94 & 0.14 & 2881 & 2008-2012 \\
\hline PUY & LAMP & surface-insitu & 45.77 & 2.97 & 1467 & 2008-2012 \\
\hline $\mathrm{ARH}$ & NIWA & surface-flask & -77.83 & 166.66 & 189 & $2000-2017$ \\
\hline BHD & NIWA, NOAA & surface-flask & -41.41 & 174.87 & 95 & $2000-2017$ \\
\hline AMT & NOAA & surface-flask & 45.03 & -68.68 & 157 & 2003-2008 \\
\hline AMY & NOAA & surface-flask & 36.54 & 126.33 & 125 & 2013-2017 \\
\hline ASC & NOAA & surface-flask & -7.97 & -14.40 & 91 & $2000-2017$ \\
\hline ASK & NOAA & surface-flask & 23.27 & 5.63 & 2715 & $2000-2017$ \\
\hline AZR & NOAA & surface-flask & 38.77 & -27.38 & 24 & $2000-2017$ \\
\hline BAL & NOAA & surface-flask & 55.50 & 16.67 & 28 & $2000-2011$ \\
\hline $\mathrm{BKT}$ & NOAA & surface-flask & -0.20 & 100.32 & 875 & 2001-2017 \\
\hline $\mathrm{BME}$ & NOAA & surface-flask & 32.37 & -64.65 & 17 & $2000-2010$ \\
\hline BMW & NOAA & surface-flask & 32.27 & -64.88 & 30 & $2000-2017$ \\
\hline BRW & NOAA & surface-flask & 71.32 & -156.61 & 11 & $2000-2017$ \\
\hline
\end{tabular}




\begin{tabular}{cc} 
BSC & NOAA \\
CBA & NOAA \\
CHR & NOAA \\
CPT & NOAA \\
CRZ & NOAA \\
EIC & NOAA \\
GMI & NOAA \\
HBA & NOAA \\
HPB & NOAA \\
HUN & NOAA \\
ICE & NOAA \\
IZO & NOAA \\
KEY & NOAA \\
KUM & NOAA \\
KZD & NOAA \\
KZM & NOAA \\
LEF & NOAA \\
LLB & NOAA \\
LLN & NOAA \\
LMP & NOAA \\
MEX & NOAA \\
MID & NOAA \\
MKN & NOAA \\
NAT & NOAA \\
NMB & NOAA \\
NWR & NOAA \\
OXK & NOAA \\
PAL & NOAA \\
PSA & NOAA \\
PTA & NOAA \\
RPB & NOAA \\
SDZ & NOAA \\
SEY & NOAA \\
SGP & NOAA \\
SHM & NOAA \\
SMO & NOAA \\
STM & NOAA \\
SUM & NOAA \\
SYO & NOAA \\
TAP & NOAA \\
THD & NOAA \\
TIK & NOAA \\
USH & NOAA \\
UTA & NOAA \\
UUM & NOAA \\
WIS & NOAA \\
WKT & NOAA \\
WLG & NOAA \\
ZEP & NOAA \\
TAC & NOAA, \\
KMW & UNIVBIS \\
SNB & UBAA \\
NGL & UBAG \\
SSL & UBAG \\
UBAG \\
\hline HS &
\end{tabular}

surface-flask

surface-flask

surface-flask

surface-flask

surface-flask

surface-flask

surface-flask

surface-flask

surface-flask

surface-flask

surface-flask

surface-flask

surface-flask

surface-flask

surface-flask

surface-flask

surface-flask

surface-flask

surface-flask

surface-flask

surface-flask

surface-flask

surface-flask

surface-flask

surface-flask

surface-flask

surface-flask

surface-flask

surface-flask

surface-flask

surface-flask

surface-flask

surface-flask

surface-flask

surface-flask

surface-flask

surface-flask

surface-flask

surface-flask

surface-flask

surface-flask

surface-flask

surface-flask

surface-flask

surface-flask

surface-flask

surface-flask

surface-flask

surface-flask

surface-flask, surface-insitu

surface-insitu

surface-insitu

surface-insitu

surface-insitu

surface-insitu

$\begin{array}{ccc}44.17 & 28.68 & 5 \\ 55.20 & -162.72 & 25 \\ 1.70 & -157.17 & 3 \\ -34.35 & 18.49 & 260 \\ -46.43 & 51.83 & 202 \\ -27.17 & -109.42 & 41 \\ 13.43 & 144.78 & 2 \\ -75.62 & -26.18 & 35 \\ 47.80 & 11.01 & 985 \\ 46.95 & 16.65 & 344 \\ 63.40 & -20.28 & 118 \\ 28.31 & -16.50 & 2378 \\ 25.67 & -80.20 & 6 \\ 19.52 & -154.82 & 3 \\ 44.45 & 77.57 & 412 \\ 43.25 & 77.88 & 2524 \\ 45.93 & -90.27 & 868 \\ 54.95 & -112.47 & 548 \\ 23.47 & 120.87 & 2867 \\ 35.52 & 12.63 & 50 \\ 18.99 & -97.31 & 4469 \\ 28.22 & -177.37 & 4 \\ -0.06 & 37.30 & 3649 \\ -6.00 & -35.20 & 0 \\ -23.57 & 15.03 & 461 \\ 40.05 & -105.59 & 3526 \\ 50.03 & 11.81 & 1185 \\ 67.97 & 24.12 & 570 \\ -64.77 & -64.05 & 15 \\ 38.95 & -123.73 & 22 \\ 13.17 & -59.43 & 20 \\ 40.65 & 117.12 & 298 \\ -4.67 & 55.17 & 3 \\ 36.60 & -97.50 & 318 \\ 52.72 & 174.10 & 28 \\ -14.25 & -170.56 & 77 \\ 66.00 & 2.00 & 4 \\ 72.58 & -38.48 & 3215 \\ -69.01 & 39.58 & 18 \\ 36.73 & 126.13 & 21 \\ 41.05 & -124.15 & 112 \\ 71.59 & 128.92 & 29 \\ -54.85 & -68.31 & 18 \\ 39.90 & -113.72 & 1332 \\ 44.45 & 111.10 & 1012 \\ 31.13 & 34.88 & 400 \\ 31.32 & -97.62 & 723 \\ 36.29 & 100.90 & 3810 \\ 78.91 & 11.89 & 479 \\ 52.52 & 1.14 & 196 \\ 53.33 & 6.27 & 4 \\ 47.05 & 12.96 & 3111 \\ 53.14 & 13.03 & 62 \\ 47.90 & 7.92 & 2671 \\ 47.42 & 10.98 & \end{array}$

2000-2011

2000-2017 except 2008

2000-2017

2000-2017

2000-2017

2000-2017

2000-2017

2000-2017

2000-2017

2000-2017

2000-2017

2000-2017

2000-2017

2000-2017

2000-2009

2000-2009

2000-2017

2007-2017

2006-2017

2006-2017

2009-2017

2000-2017

2002-2006, 2008-2011 2010-2017

2000, 2001, 2006-2017 2000-2017

2003, 2006-2017

2001-2017

2000-2017

2000-2011

2000-2017

2009-2015

2000-2017

2002-2017

2000-2017

2000-2017

2000-2009

2000-2017

2000-2017

2000-2017

2002-2017

2011-2017

2000-2017

2000-2017

2000-2017

2000-2017

2001-2010

2000-2017

2000-2017

2012, 2014-2016

2000-2012

2002-2017

2006-2013

2001-2013

2007-2017 


\begin{tabular}{ccccccc} 
ZUG & UBAG & surface-insitu & 47.42 & 10.99 & 2966 & $2000-2001$ \\
GLH & UMLT & surface-insitu & 36.07 & 14.22 & 174 & 2000, 2001, 2003, 2004, \\
CMN & UNIURB & surface-insitu & 44.17 & 10.68 & 2172 & $2007,2012-2016$ \\
CVO & UYRK & surface-insitu & 16.86 & -24.87 & 20 & $2007-2017$ \\
PDI & VNMHA & surface-insitu & 21.57 & 103.52 & 1478 & $2014-2016$ \\
\hline
\end{tabular}


Table S3. The TCCON sites used for evaluating modelled column-averaged dry-air mole fractions of CO.

\begin{tabular}{|c|c|c|c|c|c|c|}
\hline Site & Detectors & $\begin{array}{c}\text { Latitude } \\
\left({ }^{\circ}\right)\end{array}$ & $\begin{array}{c}\text { Longitude } \\
\left({ }^{\circ}\right)\end{array}$ & $\begin{array}{l}\text { Measurement } \\
\text { altitude (m) }\end{array}$ & $\begin{array}{c}\text { Data } \\
\text { period }\end{array}$ & Reference \\
\hline Indianapolis, IN, USA & xInGaAs Si & 39.86 & -86.00 & 270 & 2012 & Iraci et al., $2017 \mathrm{a}$ \\
\hline Manaus, Brazil & xInGaAs Si InSb & -3.21 & -60.60 & 50 & 2014,2015 & Dubey et al., 2017a \\
\hline Sodankylä, Finland & xInGaAs Si InSb & 67.37 & 26.63 & 188 & 2009-2017 & Kivi et al., 2017 \\
\hline Lauder, New Zealand-1 & $\begin{array}{c}\text { xInGaAs InSb } \\
\text { HgCdTe }\end{array}$ & -45.04 & 169.68 & 370 & 2004-2010 & Sherlock et al., 2017a \\
\hline Lauder, New Zealand-2 & $\begin{array}{c}\text { xInGaAs Si InSb } \\
\text { HgCdTe }\end{array}$ & -45.04 & 169.68 & 370 & 2010-2017 & Sherlock et al., 2017b \\
\hline Burgos, Philippines & xInGaAs InSb Si & 18.53 & 120.65 & 35 & 2017 & Morino et al., 2018 \\
\hline Ascension Island & xInGaAs Si & -7.92 & -14.33 & 10 & $2012-2017$ & Feist et al., 2017 \\
\hline Réunion Island & $\begin{array}{c}\text { xInGaAs Si InSb } \\
\text { MCT }\end{array}$ & -20.90 & 55.49 & 87 & 2011-2017 & De Mazière et al., 2017 \\
\hline Caltech, USA & xInGaAs Si InSb & 34.14 & -118.13 & 230 & 2012-2017 & Wennberg et al., 2017a \\
\hline Zugspitze, Germany & $\begin{array}{c}\text { xInGaAs Si InSb } \\
\mathrm{HgCdTe}\end{array}$ & 47.42 & 10.98 & 2960 & 2015-2017 & $\begin{array}{c}\text { Sussmann and } \\
\text { Rettinger, } 2018\end{array}$ \\
\hline $\begin{array}{l}\text { Ny Ålesund, } \\
\text { Spitsbergen }\end{array}$ & xInGaAs & 78.92 & 11.92 & 20 & 2006-2017 & Notholt et al., 2017a \\
\hline Orléans, France & xInGaAs Si & 47.97 & 2.11 & 130 & 2009-2017 & Warneke et al., 2017 \\
\hline $\begin{array}{c}\text { JPL, Pasadena, CA, } \\
\text { USA-1 }\end{array}$ & xInGaAs Si & 34.20 & -118.18 & 390 & 2007,2008 & Wennberg et al., 2017b \\
\hline $\begin{array}{c}\text { JPL, Pasadena, CA, } \\
\text { USA-2 }\end{array}$ & xInGaAs Si & 34.20 & -118.18 & 390 & 2011-2017 & Wennberg et al., 2017c \\
\hline Saga, Japan & $\begin{array}{c}\text { xInGaAs Si InSb } \\
\text { HgCdTe }\end{array}$ & 33.24 & 130.29 & 7 & 2011-2017 & Kawakami et al., 2017 \\
\hline Izana, Tenerife & $\begin{array}{c}\text { xInGaAs InSb } \\
\text { HgCdTe }\end{array}$ & 28.30 & -16.48 & 2370 & 2007-2017 & $\begin{array}{l}\text { Blumenstock et al., } \\
2017\end{array}$ \\
\hline Edwards, USA & xInGaAs Si & 34.96 & -117.88 & 699 & 2013-2016 & Iraci et al., 2017b \\
\hline Garmisch, Germany & xInGaAs Si InSb & 47.48 & 11.06 & 740 & 2007-2017 & $\begin{array}{c}\text { Sussmann and } \\
\text { Rettinger, } 2017\end{array}$ \\
\hline Bremen, Germany & xInGaAs & 53.10 & 8.85 & 27 & $2007-2017$ & Notholt et al., 2017b \\
\hline Karlsruhe, Germany & xInGaAs InSb & 49.10 & 8.44 & 116 & $2010-2017$ & Hase et al., 2017 \\
\hline Four Corners, USA & xInGaAs Si InSb & 36.80 & -108.48 & 1643 & 2013 & Dubey et al., 2017b \\
\hline Wollongong, Australia & xInGaAs InSb Si & -34.41 & 150.88 & 30 & $2008-2017$ & Griffith et al., 2017a \\
\hline $\begin{array}{c}\text { East Trout Lake, } \\
\text { Canada }\end{array}$ & $\mathrm{xInGaAs} \operatorname{InSb}$ & 54.36 & -104.99 & 502 & 2016,2017 & Wunch et al., 2017 \\
\hline
\end{tabular}




\begin{tabular}{|c|c|c|c|c|c|c|}
\hline Paris, France & $\begin{array}{c}\text { xInGaAs InSb } \\
\text { HgCdTe }\end{array}$ & 48.85 & 2.36 & 60 & 2014-2017 & Té et al., 2017 \\
\hline Anmeyondo, Korea & xInGaAs Si & 36.54 & 126.33 & 30 & 2015,2016 & Goo et al., 2017 \\
\hline Park Falls, WI (USA) & xInGaAs Si & 45.94 & -90.27 & 440 & 2004-2017 & Wennberg et al., 2017d \\
\hline Lamont, OK (USA) & xInGaAs Si & 36.60 & -97.49 & 320 & 2008-2017 & Wennberg et al., 2017e \\
\hline Bialystok, Poland & xInGaAs Si & 53.23 & 23.02 & 180 & 2009-2017 & Deutscher et al., 2017 \\
\hline Rikubetsu, Japan & $\begin{array}{c}\text { xInGaAs Si InSb } \\
\text { HgCdTe }\end{array}$ & 43.46 & 143.77 & 361 & 2013-2017 & Morino et al., 2017a \\
\hline Eureka, Canada & $\begin{array}{c}\text { xInGaAs InSb } \\
\text { HgCdTe }\end{array}$ & 80.05 & -86.42 & 610 & 2010-2017 & Strong et al., 2018 \\
\hline Tsukuba, Japan & $\begin{array}{c}\text { xInGaAs Si InSb } \\
\text { HgCdTe }\end{array}$ & 36.05 & 140.12 & 30 & 2011-2017 & Morino et al., 2017b \\
\hline Darwin, Australia & xInGaAs Si InSb & -12.43 & 130.89 & 30 & 2005-2017 & Griffith et al., 2017b \\
\hline
\end{tabular}




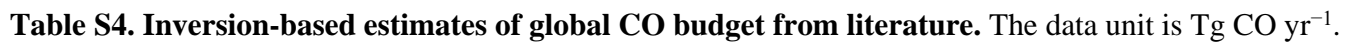

\begin{tabular}{|c|c|c|c|c|c|c|c|c|c|c|c|c|c|}
\hline Study & Constraint & Period $^{\mathrm{a}}$ & Run $^{\mathrm{b}}$ & $\mathrm{A}^{\mathrm{c}}$ & $\mathrm{BB}^{\mathrm{d}}$ & $\mathrm{O}^{\mathrm{e}}$ & $B^{f}$ & $\mathrm{DE}^{\mathrm{g}}$ & $\mathrm{CH}_{4}{ }^{\mathrm{h}}$ & VOC $^{\mathrm{i}}$ & $\mathrm{C}^{\mathrm{j}}$ & Source $^{\mathrm{k}}$ & Sink $^{1}$ \\
\hline \multirow{5}{*}{$\begin{array}{l}\text { Müller et } \\
\text { al. (2018) }\end{array}$} & IASI & 2013 & STD & 511 & 320 & $98^{\mathrm{m}}$ & & 929 & 718 & 733 & 1451 & 2381 & \\
\hline & & & $\mathrm{HN}$ & 617 & 352 & 107 & & 1076 & 820 & 815 & 1635 & 2711 & \\
\hline & & & LN & 436 & 294 & 91 & & 821 & 644 & 674 & 1318 & 2139 & \\
\hline & & & HS & 564 & 354 & 108 & & 1026 & 816 & 813 & 1629 & 2655 & \\
\hline & & & LS & 478 & 293 & 90 & & 861 & 648 & 671 & 1319 & 2180 & \\
\hline \multirow{3}{*}{$\begin{array}{c}\text { Jiang et al. } \\
(2017)\end{array}$} & MOPITT v6 & $2001-2015$ & Column & 499 & 287 & & & & & & & & \\
\hline & & & Profile & 541 & 322 & & & & & & & & \\
\hline & & & $\begin{array}{l}\text { Lower } \\
\text { profile }\end{array}$ & 581 & 345 & & & & & & & & \\
\hline $\begin{array}{l}\text { Gaubert et } \\
\text { al. (2017) }\end{array}$ & MOPITT v5 & $2002-2013$ & Reanalysis & & & & & & 782 & 468 & 1250 & & 2226 \\
\hline \multirow{2}{*}{$\begin{array}{c}\text { Yin et al. } \\
(2015)\end{array}$} & MOPITT v6 & $2002-2011$ & TransCom & & & & & 1444 & & & 1199 & 2642 & 2668 \\
\hline & & & INCA & & & & & 1540 & & & 1226 & 2765 & 2794 \\
\hline $\begin{array}{c}\text { Park et al. } \\
(2015) \\
\end{array}$ & $\delta^{18} \mathrm{O}$ & 2004 & & 716 & 377 & 20 & 97 & 1210 & 919 & 454 & 1373 & 2583 & \\
\hline $\begin{array}{l}\text { Fortems- } \\
\text { Cheiney et } \\
\text { al. (2012) }\end{array}$ & $\begin{array}{c}\text { OMI, } \\
\text { MOPITT v4, } \\
\text { WDCGG }\end{array}$ & $2005-2010$ & & & & & & 1434 & & & 1101 & 2535 & 2546 \\
\hline $\begin{array}{l}\text { Fortems- } \\
\text { Cheiney et } \\
\text { al. (2011) }\end{array}$ & MOPITT v4 & $2000-2009$ & & & & & & 1412 & & & & & \\
\hline $\begin{array}{l}\text { Kopacz et } \\
\text { al. (2010) }\end{array}$ & $\begin{array}{l}\text { Multiple } \\
\text { satellites }\end{array}$ & $\begin{array}{l}\text { MAY } 2004 \text { to } \\
\text { APR } 2005\end{array}$ & & & & & & 1350 & & & 1507 & 2857 & \\
\hline \multirow{4}{*}{$\begin{array}{c}\text { Stavrakou } \\
\text { and Müller } \\
(2006)\end{array}$} & MOPITT v3 & MAY 2000 to & GFED-Grid & 664 & 450 & 20 & 199 & 1333 & 761 & 813 & 1574 & 2907 & \\
\hline & & APR 2001 & $\begin{array}{l}\text { GFED- } \\
\text { Region- } \\
\text { Monthly }\end{array}$ & 685 & 434 & 20 & 200 & 1339 & 772 & 794 & 1566 & 2905 & \\
\hline & & & $\begin{array}{l}\text { GFED- } \\
\text { Region- } \\
\text { Annual }\end{array}$ & 675 & 412 & 20 & 186 & 1293 & 770 & 798 & 1568 & 2861 & \\
\hline & & & POET-Grid & 688 & 404 & 20 & 195 & 1307 & 755 & 798 & 1553 & 2860 & \\
\hline $\begin{array}{l}\text { Arellano et } \\
\text { al. (2006) }\end{array}$ & MOPITT v3 & $\begin{array}{l}\text { APR } 2000 \text { to } \\
\text { MAR } 2001\end{array}$ & & 841 & 563 & & & & & 394 & & & \\
\hline \multirow[t]{3}{*}{$\begin{array}{l}\text { Arellano et } \\
\text { al. (2004) }\end{array}$} & MOPITT v3 & 2000 & Column & $\begin{array}{c}844- \\
923\end{array}$ & $\begin{array}{c}508- \\
579\end{array}$ & & & & 767 & $\begin{array}{l}175- \\
209\end{array}$ & & 2388 & \\
\hline & & & $500 \mathrm{mb}$ & $\begin{array}{c}782- \\
899\end{array}$ & $\begin{array}{c}531- \\
633\end{array}$ & & & & 767 & $\begin{array}{c}148- \\
192\end{array}$ & & 2363 & \\
\hline & & & $700 \mathrm{mb}$ & $\begin{array}{c}884- \\
960\end{array}$ & $\begin{array}{l}486- \\
573\end{array}$ & & & & 767 & $\begin{array}{c}207- \\
235\end{array}$ & & 2450 & \\
\hline $\begin{array}{l}\text { Pétron et } \\
\text { al. (2004) }\end{array}$ & MOPITT & $\begin{array}{l}\text { APR } 2000 \text { to } \\
\text { MAR } 2001\end{array}$ & & 683 & 408 & & 183 & 1274 & & & 1650 & 2924 & 2630 \\
\hline
\end{tabular}

a: Year of the data. If data of multiple years are provided, the annual average are presented in the table.

b: The label of inversion run defined in each paper.

c: Anthropogenic emissions; ${ }^{\mathrm{d}}$ : Biomass burning emissions; e: Oceanic emissions; ${ }^{\mathrm{f}}$ : Biogenic emissions; ${ }^{\mathrm{g}}$ : surface direct emissions from

5 the sum of anthropogenic, biomass burning, oceanic, and biogenic sources; ${ }^{\text {h}}$ : $\mathrm{CO}$ from the oxidation of $\mathrm{CH}_{4}$; ${ }^{\mathrm{i}}$ : $\mathrm{CO}$ from the oxidation of NMVOCs; ${ }^{\mathrm{j}}$ : chemical production of $\mathrm{CO}$ from the sum of $\mathrm{CH}_{4}$ and NMVOCs oxidation; ${ }^{\mathrm{k}}$ : $\mathrm{CO}$ total source; ${ }^{1}$ : $\mathrm{CO}$ total sink; ${ }^{\mathrm{m}}$ : The sum of oceanic and soil emissions are presented in Müller et al. (2018). 
Table S5. Global atmospheric carbon monoxide budget during 2000-2017. Average CO budget $\left(10^{3} \mathrm{Tg} \mathrm{CO}^{-1}\right)$ are derived from Inversions \#1, \#2, and \#3 for the time period of 2000-2017, 2005-2017, and 2010-2017.

\begin{tabular}{|c|c|c|c|c|c|c|}
\hline Unit: $10^{3} \mathrm{Tg} \mathrm{CO} \mathrm{yr}^{-1}$ & $\begin{array}{c}\text { Inv \#1 } \\
2000-2017\end{array}$ & $\begin{array}{c}\text { Inv \#1 } \\
2005-2017\end{array}$ & $\begin{array}{c}\text { Inv \#1 } \\
2010-2017\end{array}$ & $\begin{array}{c}\text { Inv \#2 } \\
2005-2017\end{array}$ & $\begin{array}{c}\text { Inv \#2 } \\
2010-2017\end{array}$ & $\begin{array}{c}\text { Inv \#3 } \\
2010-2017\end{array}$ \\
\hline \multicolumn{7}{|l|}{ Sources } \\
\hline Anthropogenic & 0.7 & 0.7 & 0.7 & 0.7 & 0.7 & 0.7 \\
\hline Biomass burning & 0.5 & 0.4 & 0.4 & 0.4 & 0.4 & 0.4 \\
\hline Oceanic & 0.02 & 0.02 & 0.02 & 0.02 & 0.02 & 0.02 \\
\hline Biogenic & 0.2 & 0.2 & 0.2 & 0.2 & 0.2 & 0.2 \\
\hline Sub-total direct emissions & 1.4 & 1.3 & 1.3 & 1.3 & 1.3 & 1.3 \\
\hline Oxidation of $\mathrm{CH}_{4}$ & 0.9 & 0.9 & 0.9 & 0.9 & 0.9 & 0.9 \\
\hline Oxidation of NMVOCs & 0.3 & 0.3 & 0.3 & 0.3 & 0.3 & 0.3 \\
\hline Sub-total chemical production & 1.2 & 1.2 & 1.2 & 1.2 & 1.2 & 1.2 \\
\hline Total sources & 2.6 & 2.6 & 2.5 & 2.6 & 2.5 & 2.5 \\
\hline \multicolumn{7}{|l|}{ Sinks } \\
\hline $\mathrm{OH}$ reaction & 2.6 & 2.6 & 2.6 & 2.6 & 2.6 & 2.6 \\
\hline
\end{tabular}


Table S6. Global atmospheric carbon monoxide budget during 2000-2017 estimated using the prior information. Average CO


all derived from the LMDz-SACS modelling results with the prior information. Absolute and relative trends are both presented with $95 \%$ confidence limits. Significant trends are marked by asterisks $(* p<0.1, * * p<0.05$, and $* * * p<0.01)$.

\begin{tabular}{|c|c|c|c|c|}
\hline & $\begin{array}{c}\text { Average } \\
\left(10^{3} \mathrm{Tg} \mathrm{CO} \mathrm{yr}^{-1}\right)\end{array}$ & $\begin{array}{l}\mathrm{CV} \\
(\%)\end{array}$ & 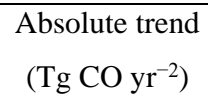 & $\begin{array}{c}\text { Relative trend } \\
\left(\% \mathrm{yr}^{-1}\right)\end{array}$ \\
\hline \multicolumn{5}{|l|}{ Sources } \\
\hline Anthropogenic & 0.6 & 2.2 & $2.0 \pm 0.9 * * *$ & $0.33 \pm 0.14 * * *$ \\
\hline Biomass burning & 0.3 & 12.3 & $-1.2 \pm 4.2$ & $-0.43 \pm 1.43$ \\
\hline Oceanic & 0.02 & 0.1 & $0.0 \pm 0.0$ & $0.00 \pm 0.01$ \\
\hline Biogenic & 0.09 & 3.3 & $0.2 \pm 0.3$ & $0.21 \pm 0.33$ \\
\hline Sub-total direct emissions & 1.1 & 3.6 & $1.0 \pm 3.9$ & $0.09 \pm 0.39$ \\
\hline Oxidation of $\mathrm{CH}_{4}$ & 0.9 & 1.6 & $2.7 \pm 0.3 * * *$ & $0.31 \pm 0.04 * * *$ \\
\hline Oxidation of NMVOCs & 0.3 & 0.2 & $-0.06 \pm 0.05^{* *}$ & $-0.02 \pm 0.02 * *$ \\
\hline Sub-total chemical oxidation & 1.2 & 1.2 & $2.6 \pm 0.3 * * *$ & $0.22 \pm 0.03^{* * *}$ \\
\hline Total sources & 2.3 & 1.9 & $3.6 \pm 3.8^{*}$ & $0.16 \pm 0.18^{*}$ \\
\hline \multicolumn{5}{|l|}{ Sinks } \\
\hline $\mathrm{OH}$ reaction & 2.3 & 1.5 & $2.4 \pm 3.2$ & $0.11 \pm 0.14$ \\
\hline
\end{tabular}


Table S7. Anthropogenic CO emissions and trends during 2000-2017 by region derived from Inversion \#1 and Inversion \#4.


$\mathrm{yr}^{-1}$ ) are estimated for each region on the basis of Inversion \#1, Inversion \#4, and the prior CEDS inventory. Absolute and relative trends are both presented with $95 \%$ confidence limits. Significant trends are marked by asterisks $(* p<0.1, * * p<0.05$, and $* * * p<0.01)$.

\begin{tabular}{|c|c|c|c|c|c|c|c|c|}
\hline & $\begin{array}{l}\text { Average } \\
(\mathrm{Tg} \mathrm{CO} \\
\left.\mathrm{yr}^{-1}\right)\end{array}$ & $\begin{array}{l}\mathrm{CV} \\
(\%)\end{array}$ & $\begin{array}{l}\text { Absolute trend } \\
\text { (Inversion \#1) } \\
\left(\mathrm{Tg} \mathrm{CO} \mathrm{yr}^{-2}\right)\end{array}$ & $\begin{array}{c}\text { Relative trend } \\
\text { (Inversion \#1) } \\
\left(\% \mathrm{yr}^{-1}\right)\end{array}$ & $\begin{array}{c}\text { Relative trend } \\
\text { (Inversion \#4) } \\
\left(\% \mathrm{yr}^{-1}\right)\end{array}$ & $\begin{array}{c}\text { Average } \\
\text { (CEDS) } \\
(\mathrm{Tg} \mathrm{CO} \\
\left.\mathrm{yr}^{-1}\right)\end{array}$ & $\begin{array}{l}\text { Absolute trend } \\
\text { (CEDS }) \\
\left(\mathrm{Tg} \mathrm{CO} \mathrm{yr}^{-2}\right)\end{array}$ & $\begin{array}{l}\text { Relative trend } \\
\qquad \begin{array}{l}\text { (CEDS }) \\
\left(\% \mathrm{yr}^{-1}\right)\end{array}\end{array}$ \\
\hline $\mathrm{CHN}$ & 183.0 & 6.4 & $-1.4 \pm 0.9 * * *$ & $-0.77 \pm 0.49 * * *$ & $-1.33 \pm 0.44 * * *$ & 185.8 & $2.2 \pm 0.7 * * *$ & $1.31 \pm 0.44 * * *$ \\
\hline SAS & 83.6 & 7.4 & $0.8 \pm 0.5 * * *$ & $1.01 \pm 0.64 * * *$ & $0.16 \pm 0.66$ & 84.8 & $2.3 \pm 0.3 * * *$ & $3.51 \pm 0.40 * * *$ \\
\hline USA & 69.9 & 26.8 & $-3.4 \pm 0.6^{* * *}$ & $-3.01 \pm 0.53 * * *$ & $-2.16 \pm 0.61 * * *$ & 59.5 & $-3.1 \pm 0.5^{* * *}$ & $-3.26 \pm 0.56 * * *$ \\
\hline EQAF & 64.9 & 10.8 & $1.1 \pm 0.4 * * *$ & $2.01 \pm 0.72 * * *$ & $1.37 \pm 0.57 * * *$ & 45.9 & $0.8 \pm 0.1 * * *$ & $2.06 \pm 0.25 * * *$ \\
\hline EU & 53.7 & 18.0 & $-1.6 \pm 0.5 * * *$ & $-2.41 \pm 0.74 * * *$ & $-1.10 \pm 0.83 * *$ & 28.9 & $-1.0 \pm 0.1 * * *$ & $-2.62 \pm 0.35 * * *$ \\
\hline SEAS & 43.8 & 25.3 & $-0.2 \pm 1.1$ & $-0.45 \pm 2.04$ & $-0.01 \pm 1.85$ & 56.3 & $1.3 \pm 0.2 * * *$ & $3.01 \pm 0.38 * * *$ \\
\hline MIDE & 37.3 & 16.8 & $-0.4 \pm 0.6$ & $-1.09 \pm 1.54$ & $0.54 \pm 1.49$ & 18.6 & $-0.05 \pm 0.1$ & $-0.31 \pm 0.61$ \\
\hline NAF & 31.2 & 14.6 & $0.7 \pm 0.3 * * *$ & $2.81 \pm 1.16^{* * *}$ & $2.39 \pm 0.66^{* * *}$ & 19.6 & $0.4 \pm 0.1 * * *$ & $2.02 \pm 0.34 * * *$ \\
\hline RUS & 28.3 & 20.3 & $-0.4 \pm 0.6$ & $-1.25 \pm 1.74$ & $-1.65 \pm 1.68^{*}$ & 11.6 & $-0.3 \pm 0.0 * * *$ & $-2.04 \pm 0.25 * * *$ \\
\hline SAF & 27.6 & 12.3 & $0.3 \pm 0.3^{*}$ & $0.84 \pm 1.00 *$ & $0.85 \pm 1.04$ & 15.7 & $0.1 \pm 0.0 * * *$ & $0.78 \pm 0.14 * * *$ \\
\hline BRA & 27.0 & 9.0 & $-0.1 \pm 0.2$ & $-0.32 \pm 0.76$ & $-0.70 \pm 0.78^{*}$ & 15.8 & $-0.05 \pm 0.06^{*}$ & $-0.33 \pm 0.36^{*}$ \\
\hline SSA & 11.8 & 21.9 & $-0.02 \pm 0.3$ & $-0.10 \pm 1.45$ & $-2.01 \pm 1.37 * * *$ & 9.0 & $0.2 \pm 0.1 * * *$ & $2.03 \pm 0.59 * * *$ \\
\hline KAJ & 11.7 & 23.8 & $-0.5 \pm 0.2 * * *$ & $-3.02 \pm 0.97 * * *$ & $-1.59 \pm 0.85^{* * *}$ & 13.3 & $-0.4 \pm 0.0^{* * *}$ & $-2.24 \pm 0.22 * * *$ \\
\hline CAS & 11.5 & 16.5 & $0.03 \pm 0.2$ & $0.34 \pm 2.26$ & $1.03 \pm 1.86$ & 5.4 & $0.05 \pm 0.04 * *$ & $1.12 \pm 0.83 * *$ \\
\hline CAM & 11.5 & 32.0 & $-0.4 \pm 0.3 * * *$ & $-2.09 \pm 1.40 * * *$ & $-1.96 \pm 1.34 * * *$ & 18.3 & $-0.2 \pm 0.0 * * *$ & $-0.83 \pm 0.17 * * *$ \\
\hline CAN & 9.6 & 39.6 & $0.2 \pm 0.4$ & $2.03 \pm 3.36$ & $-4.09 \pm 2.25 * * *$ & 5.8 & $-0.2 \pm 0.0 * * *$ & $-2.78 \pm 0.38 * * *$ \\
\hline NSA & 8.6 & 13.7 & $-0.01 \pm 0.1$ & $-0.07 \pm 1.22$ & $-0.89 \pm 1.15$ & 11.3 & $0.01 \pm 0.06$ & $0.11 \pm 0.56$ \\
\hline OCE & 8.0 & 28.6 & $-0.1 \pm 0.2$ & $-1.21 \pm 2.69$ & $-0.98 \pm 1.75$ & 3.9 & $-0.03 \pm 0.02 * * *$ & $-0.82 \pm 0.47 * * *$ \\
\hline
\end{tabular}


Table S8. Anthropogenic CO emissions and trends during 2005-2017 by region derived from Inversion \#2. Average anthropogenic

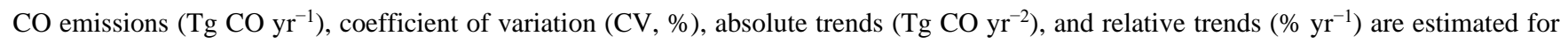
each region on the basis of Inversion \#2 and the prior CEDS inventory. Absolute and relative trends are both presented with $95 \%$ confidence limits. Significant trends are marked by asterisks $(* p<0.1, * * p<0.05$, and $* * * p<0.01)$.

\begin{tabular}{|c|c|c|c|c|c|c|c|}
\hline &  & $\begin{array}{l}\mathrm{CV} \\
(\%)\end{array}$ & $\begin{array}{l}\text { Absolute trend } \\
\text { (Inversion \#2) } \\
\left(\mathrm{Tg} \mathrm{CO} \mathrm{yr}{ }^{-2}\right)\end{array}$ & $\begin{array}{c}\text { Relative trend } \\
\text { (Inversion \#2) } \\
\left(\% \mathrm{yr}^{-1}\right)\end{array}$ & $\begin{array}{c}\text { Average } \\
(\mathrm{CEDS}) \\
\left(\mathrm{Tg} \mathrm{CO} \mathrm{yr}^{-1}\right)\end{array}$ &  & $\begin{array}{l}\text { Relative trend } \\
\qquad(\mathrm{CEDS}) \\
\left(\% \mathrm{yr}^{-1}\right)\end{array}$ \\
\hline $\mathrm{CHN}$ & 176.2 & 10.5 & $-4.1 \pm 1.9 * * *$ & $-2.04 \pm 0.94 * * *$ & 193.4 & $0.9 \pm 0.9 *$ & $0.48 \pm 0.49 *$ \\
\hline SAS & 85.9 & 5.2 & $0.0 \pm 0.8$ & $0.01 \pm 0.90$ & 90.9 & $2.0 \pm 0.5^{* * *}$ & $2.66 \pm 0.62 * * *$ \\
\hline USA & 61.1 & 22.8 & $-3.4 \pm 1.0 * * *$ & $-3.96 \pm 1.11 * * *$ & 50.5 & $-2.2 \pm 0.6^{* * *}$ & $-3.15 \pm 0.89 * * *$ \\
\hline EQAF & 69.7 & 6.3 & $0.8 \pm 0.6^{* *}$ & $1.14 \pm 0.91 * *$ & 47.9 & $0.9 \pm 0.2 * * *$ & $2.13 \pm 0.43 * * *$ \\
\hline $\mathrm{EU}$ & 53.7 & 23.3 & $-2.2 \pm 1.6^{* *}$ & $-3.45 \pm 2.53 * *$ & 26.0 & $-0.8 \pm 0.2 * * *$ & $-2.41 \pm 0.56 * * *$ \\
\hline SEAS & 30.7 & 54.7 & $0.0 \pm 3.0$ & $0.07 \pm 7.58$ & 60.1 & $1.1 \pm 0.3^{* * *}$ & $2.10 \pm 0.51 * * *$ \\
\hline MIDE & 41.6 & 28.9 & $-1.6 \pm 1.9^{*}$ & $-3.62 \pm 4.23^{*}$ & 18.8 & $-0.3 \pm 0.1 * * *$ & $-1.41 \pm 0.38 * * *$ \\
\hline NAF & 36.0 & 18.3 & $0.2 \pm 1.2$ & $0.87 \pm 4.07$ & 20.5 & $0.4 \pm 0.1 * * *$ & $2.11 \pm 0.63 * * *$ \\
\hline RUS & 28.0 & 20.9 & $-0.3 \pm 1.0$ & $-0.87 \pm 3.20$ & 10.8 & $-0.3 \pm 0.1 * * *$ & $-2.00 \pm 0.48 * * *$ \\
\hline SAF & 29.1 & 14.6 & $-0.1 \pm 0.7$ & $-0.50 \pm 3.02$ & 16.0 & $0.1 \pm 0.0^{* * *}$ & $0.90 \pm 0.26 * * *$ \\
\hline BRA & 28.9 & 11.6 & $-0.2 \pm 0.6$ & $-0.92 \pm 2.10$ & 15.7 & $-0.1 \pm 0.1 * * *$ & $-0.85 \pm 0.41 * * *$ \\
\hline SSA & 13.2 & 19.4 & $0.0 \pm 0.5$ & $0.30 \pm 3.59$ & 9.4 & $0.2 \pm 0.1 * * *$ & $2.76 \pm 0.97 * * *$ \\
\hline KAJ & 9.4 & 32.6 & $-0.5 \pm 0.4^{* *}$ & $-3.22 \pm 3.05^{* *}$ & 12.5 & $-0.4 \pm 0.1 * * *$ & $-2.62 \pm 0.42 * * *$ \\
\hline CAS & 12.1 & 23.8 & $-0.3 \pm 0.5$ & $-1.88 \pm 3.04$ & 5.6 & $0.0 \pm 0.1$ & $-0.25 \pm 1.12$ \\
\hline CAM & 4.0 & 150.8 & $0.4 \pm 1.0$ & $8.05 \pm 19.37$ & 17.8 & $-0.1 \pm 0.0^{* * *}$ & $-0.55 \pm 0.16^{* * *}$ \\
\hline CAN & 12.0 & 41.9 & $0.4 \pm 0.9$ & $4.93 \pm 10.19$ & 5.1 & $-0.2 \pm 0.0^{* * *}$ & $-2.64 \pm 0.63 * * *$ \\
\hline NSA & 5.1 & 63.2 & $0.2 \pm 0.6$ & $2.83 \pm 9.80$ & 11.4 & $-0.1 \pm 0.1$ & $-0.60 \pm 0.96$ \\
\hline OCE & 8.5 & 25.2 & $-0.4 \pm 0.3^{* *}$ & $-4.33 \pm 3.15^{* *}$ & 3.8 & $-0.1 \pm 0.0^{* * *}$ & $-1.47 \pm 0.4 * * *$ \\
\hline
\end{tabular}


Table S9. Anthropogenic CO emissions and trends during 2010-2017 by region derived from Inversion \#3. Average anthropogenic


each region on the basis of Inversion \#3 and the prior CEDS inventory. Absolute and relative trends are both presented with 95\% confidence limits. Significant trends are marked by asterisks $(* p<0.1, * * p<0.05$, and $* * * p<0.01)$.

\begin{tabular}{|c|c|c|c|c|c|c|c|}
\hline &  & $\begin{array}{l}\mathrm{CV} \\
(\%)\end{array}$ & $\begin{array}{c}\text { Absolute trend } \\
\text { (Inversion \#3) } \\
\left(\mathrm{Tg} \mathrm{CO} \mathrm{yr}{ }^{-2}\right)\end{array}$ & $\begin{array}{c}\text { Relative trend } \\
\text { (Inversion \#3) } \\
\left(\% \mathrm{yr}^{-1}\right)\end{array}$ & $\begin{array}{c}\text { Average } \\
(\mathrm{CEDS}) \\
\left(\mathrm{Tg} \mathrm{CO} \mathrm{yr}{ }^{-1}\right)\end{array}$ & $\begin{array}{l}\text { Absolute trend } \\
\text { (CEDS }) \\
\left(\mathrm{Tg} \mathrm{CO} \mathrm{yr}^{-2}\right)\end{array}$ & $\begin{array}{l}\text { Relative trend } \\
\qquad(\mathrm{CEDS}) \\
\left(\% \mathrm{yr}^{-1}\right)\end{array}$ \\
\hline $\mathrm{CHN}$ & 162.2 & 9.2 & $-3.7 \pm 5.3$ & $-2.23 \pm 3.16$ & 195.5 & $-0.3 \pm 0.3 *$ & $-0.14 \pm 0.17^{*}$ \\
\hline SAS & 87.4 & 4.2 & $-1.1 \pm 1.2 *$ & $-1.17 \pm 1.31^{*}$ & 96.6 & $0.9 \pm 0.3 * * *$ & $0.94 \pm 0.36 * * *$ \\
\hline USA & 53.2 & 13.4 & $-2.2 \pm 2.2 *$ & $-3.9 \pm 4.0 *$ & 44.6 & $-1.0 \pm 0.4^{* * *}$ & $-2.01 \pm 0.83 * * *$ \\
\hline EQAF & 73.3 & 5.9 & $0.9 \pm 1.6$ & $1.24 \pm 2.24$ & 50.4 & $0.5 \pm 0.3 * * *$ & $0.99 \pm 0.61 * * *$ \\
\hline $\mathrm{EU}$ & 50.6 & 17.2 & $-1.6 \pm 3.4$ & $-2.88 \pm 6.00$ & 23.9 & $-0.5 \pm 0.3 * * *$ & $-1.74 \pm 1.02 * * *$ \\
\hline SEAS & 30.9 & 27.7 & $-0.6 \pm 3.7$ & $-1.61 \pm 10.74$ & 62.9 & $0.6 \pm 0.3 * * *$ & $0.92 \pm 0.50 * * *$ \\
\hline MIDE & 40.8 & 25.6 & $-2.3 \pm 4.0$ & $-4.20 \pm 7.32$ & 17.9 & $-0.1 \pm 0.1 * *$ & $-0.66 \pm 0.52 * *$ \\
\hline NAF & 38.7 & 12.8 & $-0.6 \pm 2.1$ & $-1.36 \pm 5.14$ & 21.5 & $0.2 \pm 0.1 * * *$ & $0.77 \pm 0.38 * * *$ \\
\hline RUS & 28.8 & 18.7 & $-0.6 \pm 2.3$ & $-1.49 \pm 6.06$ & 10.1 & $-0.1 \pm 0.0^{* * *}$ & $-1.15 \pm 0.46 * * *$ \\
\hline SAF & 29.6 & 10.9 & $-0.7 \pm 1.2$ & $-1.89 \pm 3.26$ & 16.3 & $0.2 \pm 0.1 * * *$ & $1.15 \pm 0.72 * * *$ \\
\hline BRA & 28.6 & 7.1 & $0.2 \pm 0.9$ & $0.84 \pm 3.33$ & 15.3 & $0.0 \pm 0.0 * *$ & $-0.16 \pm 0.16 * *$ \\
\hline SSA & 13.3 & 22.5 & $-0.4 \pm 1.2$ & $-3.09 \pm 8.62$ & 10.0 & $0.1 \pm 0.0 * *$ & $0.57 \pm 0.51 * *$ \\
\hline KAJ & 7.9 & 19.5 & $-0.1 \pm 0.7$ & $-1.31 \pm 8.48$ & 11.4 & $-0.3 \pm 0.1 * * *$ & $-2.14 \pm 1.06 * * *$ \\
\hline CAS & 11.3 & 16.8 & $-0.1 \pm 0.8$ & $-1.00 \pm 5.64$ & 5.5 & $0.0 \pm 0.0$ & $-0.34 \pm 0.63$ \\
\hline CAM & 4.1 & 102.9 & $-0.1 \pm 1.9$ & $-11.33 \pm 281.09$ & 17.5 & $-0.1 \pm 0.1 * *$ & $-0.35 \pm 0.35 * *$ \\
\hline $\mathrm{CAN}$ & 12.6 & 35.4 & $0.7 \pm 1.8$ & $6.07 \pm 16.29$ & 4.6 & $-0.1 \pm 0.1 * *$ & $-1.81 \pm 1.30 * *$ \\
\hline NSA & 4.8 & 62.8 & $0.1 \pm 1.3$ & $2.19 \pm 24.06$ & 11.3 & $-0.2 \pm 0.2 * *$ & $-1.82 \pm 1.65 * *$ \\
\hline OCE & 8.1 & 20.7 & $-0.5 \pm 0.6^{*}$ & $-6.02 \pm 6.98^{*}$ & 3.7 & $0.0 \pm 0.0 * * *$ & $-1.24 \pm 0.51 * * *$ \\
\hline
\end{tabular}


Table S10. Biomass burning CO emissions and trends during 2000-2017 by region derived from Inversion \#1 and Inversion \#4.

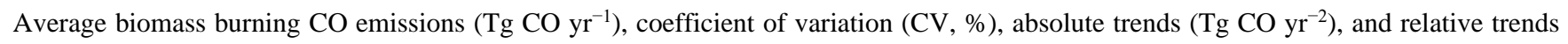
$\left(\% \mathrm{yr}^{-1}\right)$ are estimated for each region on the basis of Inversion \#1, Inversion \#4, and the prior GFED 4.1s inventory. Absolute and relative trends are both presented with $95 \%$ confidence limits. Significant trends are marked by asterisks $(* p<0.1, * * p<0.05$, and $* * * p<0.01)$.

\begin{tabular}{|c|c|c|c|c|c|c|c|c|}
\hline & $\begin{array}{c}\text { Average } \\
(\mathrm{Tg} \mathrm{CO} \\
\left.\mathrm{yr}^{-1}\right)\end{array}$ & $\begin{array}{l}\mathrm{CV} \\
(\%)\end{array}$ & $\begin{array}{c}\text { Absolute trend } \\
\text { (Inversion \#1) } \\
\left(\mathrm{Tg} \mathrm{CO} \mathrm{yr}^{-2}\right)\end{array}$ & $\begin{array}{c}\text { Relative trend } \\
\text { (Inversion \#1) } \\
\left(\% \mathrm{yr}^{-1}\right)\end{array}$ & $\begin{array}{c}\text { Relative trend } \\
\text { (Inversion \#4) } \\
\left(\% \mathrm{yr}^{-1}\right)\end{array}$ & $\begin{array}{c}\text { Average } \\
\text { (GFED) } \\
(\mathrm{Tg} \mathrm{CO} \\
\left.\mathrm{yr}^{-1}\right)\end{array}$ & $\begin{array}{l}\text { Absolute trend } \\
(\text { GFED 4.1s) } \\
\left(\mathrm{Tg} \mathrm{CO} \mathrm{yr}^{-2}\right)\end{array}$ & $\begin{array}{l}\text { Relative trend } \\
\text { (GFED 4.1s) } \\
\left(\% \mathrm{yr}^{-1}\right)\end{array}$ \\
\hline EQAF & 92.6 & 14.2 & $-1.6 \pm 1.1 * * *$ & $-1.47 \pm 0.99 * * *$ & $-1.08 \pm 0.85^{* *}$ & 74.7 & $-1.1 \pm 0.5^{* * *}$ & $-1.27 \pm 0.55 * * *$ \\
\hline SAF & 82.8 & 10.0 & $-0.2 \pm 0.8$ & $-0.25 \pm 1.05$ & $-0.26 \pm 0.78$ & 55.0 & $0.0 \pm 0.4$ & $0.00 \pm 0.79$ \\
\hline BRA & 58.8 & 43.8 & $-0.4 \pm 2.6$ & $-1.10 \pm 7.57$ & $-1.37 \pm 4.70$ & 33.9 & $-0.1 \pm 1.8$ & $-0.53 \pm 9.97$ \\
\hline SEAS & 50.1 & 59.5 & $-0.2 \pm 3.0$ & $-0.57 \pm 11.47$ & $-1.34 \pm 5.62$ & 47.1 & $0.5 \pm 3.4$ & $2.31 \pm 16.14$ \\
\hline RUS & 37.5 & 37.4 & $-0.1 \pm 1.4$ & $-0.40 \pm 4.43$ & $-1.27 \pm 2.68$ & 34.4 & $-0.4 \pm 1.6$ & $-1.16 \pm 4.96$ \\
\hline OCE & 28.4 & 37.9 & $-0.9 \pm 1.0^{*}$ & $-2.23 \pm 2.43 *$ & $-1.53 \pm 2.74$ & 15.5 & $-0.4 \pm 0.5^{*}$ & $-2.52 \pm 2.85^{*}$ \\
\hline NAF & 27.2 & 10.0 & $0.01 \pm 0.28$ & $0.02 \pm 1.03$ & $-0.02 \pm 0.91$ & 20.9 & $-0.1 \pm 0.2$ & $-0.52 \pm 0.64$ \\
\hline SSA & 18.9 & 52.2 & $-0.7 \pm 0.9$ & $-2.69 \pm 3.64$ & $-2.94 \pm 2.59 * *$ & 11.1 & $-0.2 \pm 0.5$ & $-1.93 \pm 4.39$ \\
\hline CAN & 14.2 & 47.6 & $0.8 \pm 0.6^{* *}$ & $11.00 \pm 8.34 * *$ & $1.78 \pm 4.09$ & 14.7 & $0.8 \pm 0.7 * *$ & $15.17 \pm 12.66^{* *}$ \\
\hline USA & 6.7 & 45.4 & $0.1 \pm 0.3$ & $1.87 \pm 6.19$ & $2.80 \pm 3.17 *$ & 6.0 & $0.1 \pm 0.4$ & $1.29 \pm 9.38$ \\
\hline CAM & 6.3 & 56.9 & $-0.3 \pm 0.3$ & $-1.98 \pm 2.57$ & $-1.07 \pm 2.04$ & 5.3 & $-0.1 \pm 0.2$ & $-1.47 \pm 2.55$ \\
\hline CAS & 5.4 & 57.8 & $-0.2 \pm 0.3$ & $-6.29 \pm 7.70$ & $-3.56 \pm 3.22 * *$ & 3.1 & $-0.1 \pm 0.2$ & $-5.43 \pm 7.48$ \\
\hline EU & 4.7 & 25.4 & $-0.1 \pm 0.1 * *$ & $-1.48 \pm 1.45^{* *}$ & $-0.74 \pm 1.42$ & 2.4 & $-0.06 \pm 0.06^{* *}$ & $-1.63 \pm 1.62 * *$ \\
\hline NSA & 3.8 & 43.4 & $-0.1 \pm 0.2$ & $-2.16 \pm 3.04$ & $-2.32 \pm 2.86$ & 3.8 & $-0.08 \pm 0.12$ & $-1.63 \pm 2.53$ \\
\hline $\mathrm{CHN}$ & 3.7 & 18.7 & $0.04 \pm 0.07$ & $1.14 \pm 1.75$ & $0.92 \pm 1.59$ & 3.7 & $0.1 \pm 0.1 * *$ & $3.07 \pm 2.41 * *$ \\
\hline SAS & 2.5 & 30.3 & $0.04 \pm 0.07$ & $1.43 \pm 2.82$ & $0.87 \pm 2.36$ & 2.1 & $0.06 \pm 0.06^{* *}$ & $3.42 \pm 3.03^{* *}$ \\
\hline MIDE & 0.9 & 29.8 & $0.00 \pm 0.03$ & $0.21 \pm 3.04$ & $1.76 \pm 3.03$ & 0.3 & $0.00 \pm 0.01$ & $1.51 \pm 3.21$ \\
\hline KAJ & 0.3 & 39.8 & $-0.01 \pm 0.01 * *$ & $-2.51 \pm 2.17 * *$ & $-0.64 \pm 1.58$ & 0.3 & $-0.01 \pm 0.01$ & $-1.78 \pm 2.17$ \\
\hline
\end{tabular}


Table S11. Biomass burning CO emissions and trends during 2005-2017 by region derived from Inversion \#2. Average biomass

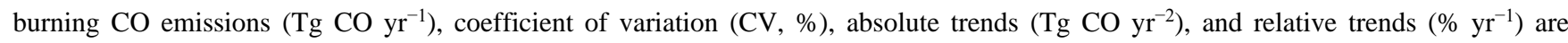
estimated for each region on the basis of Inversion \#2 and the prior GFED 4.1s inventory. Absolute and relative trends are both presented with $95 \%$ confidence limits. Significant trends are marked by asterisks $(* p<0.1, * * p<0.05$, and $* * * p<0.01)$.

\begin{tabular}{|c|c|c|c|c|c|c|c|}
\hline & 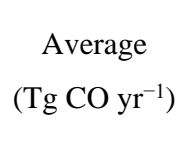 & $\begin{array}{l}\mathrm{CV} \\
(\%)\end{array}$ & $\begin{array}{l}\text { Absolute trend } \\
\text { (Inversion \#2) } \\
\left(\mathrm{Tg} \mathrm{CO} \mathrm{yr}^{-2}\right)\end{array}$ & $\begin{array}{c}\text { Relative trend } \\
\text { (Inversion \#2) } \\
\left(\% \mathrm{yr}^{-1}\right)\end{array}$ & $\begin{array}{c}\text { Average } \\
(\text { GFED } 4.1 \mathrm{~s}) \\
\left(\mathrm{Tg} \mathrm{CO} \mathrm{yr}^{-1}\right)\end{array}$ & $\begin{array}{l}\text { Absolute trend } \\
(\text { GFED 4.1s) } \\
\left(\mathrm{Tg} \mathrm{CO} \mathrm{yr}^{-2}\right)\end{array}$ & $\begin{array}{l}\text { Relative trend } \\
\text { (GFED 4.1s) } \\
\left(\% \mathrm{yr}^{-1}\right)\end{array}$ \\
\hline EQAF & 85.3 & 12.4 & $-0.7 \pm 1.8$ & $-0.71 \pm 1.73$ & 71.7 & $-0.8 \pm 0.8^{*}$ & $-0.95 \pm 0.96^{*}$ \\
\hline SAF & 83.7 & 13.1 & $-1.0 \pm 1.8$ & $-1.17 \pm 2.05$ & 55.5 & $-0.3 \pm 0.4$ & $-0.58 \pm 0.76$ \\
\hline BRA & 59.2 & 43.8 & $-1.8 \pm 4.4$ & $-1.81 \pm 4.50$ & 35.4 & $-1.5 \pm 3.4$ & $-2.47 \pm 5.62$ \\
\hline SEAS & 45.6 & 59.8 & $-0.6 \pm 4.8$ & $-1.48 \pm 11.14$ & 49.1 & $-0.6 \pm 6.3$ & $-1.35 \pm 15.51$ \\
\hline RUS & 35.9 & 34.5 & $0.5 \pm 2.2$ & $1.76 \pm 7.58$ & 32.2 & $0.3 \pm 2.1$ & $1.62 \pm 9.86$ \\
\hline OCE & 25.5 & 42.3 & $-0.5 \pm 1.9$ & $-2.97 \pm 11.84$ & 13.8 & $0.0 \pm 0.9$ & $-0.36 \pm 8.92$ \\
\hline $\mathrm{NAF}$ & 27.6 & 10.2 & $-0.2 \pm 0.5$ & $-0.53 \pm 1.62$ & 20.6 & $-0.1 \pm 0.3$ & $-0.58 \pm 1.04$ \\
\hline SSA & 18.0 & 47.5 & $-1.0 \pm 1.4$ & $-4.14 \pm 5.49$ & 10.8 & $-0.5 \pm 0.9$ & $-3.44 \pm 5.56$ \\
\hline $\mathrm{CAN}$ & 16.1 & 51.8 & $1.2 \pm 1.2 *$ & $11.78 \pm 12.07 *$ & 15.9 & $1.2 \pm 1.1 * *$ & $10.55 \pm 10.00 * *$ \\
\hline USA & 7.0 & 38.5 & $0.0 \pm 0.5$ & $0.31 \pm 4.85$ & 5.9 & $0.0 \pm 0.6$ & $0.15 \pm 5.36$ \\
\hline CAM & 4.9 & 54.7 & $-0.2 \pm 0.4$ & $-2.35 \pm 4.36$ & 5.0 & $-0.2 \pm 0.3$ & $-2.07 \pm 3.27$ \\
\hline CAS & 5.2 & 42.7 & $-0.3 \pm 0.3^{*}$ & $-4.83 \pm 5.18 *$ & 2.8 & $-0.2 \pm 0.2 *$ & $-6.88 \pm 8.18^{*}$ \\
\hline EU & 5.1 & 29.4 & $-0.1 \pm 0.3$ & $-1.99 \pm 4.50$ & 2.3 & $-0.1 \pm 0.1$ & $-1.91 \pm 3.40$ \\
\hline NSA & 2.2 & 62.7 & $0.1 \pm 0.2$ & $3.41 \pm 8.79$ & 3.4 & $0.0 \pm 0.2$ & $0.38 \pm 5.28$ \\
\hline $\mathrm{CHN}$ & 3.7 & 16.0 & $0.0 \pm 0.1$ & $-0.26 \pm 2.87$ & 4.0 & $0.1 \pm 0.1$ & $2.27 \pm 4.08$ \\
\hline SAS & 2.6 & 31.6 & $0.0 \pm 0.1$ & $-0.30 \pm 5.84$ & 2.3 & $0.0 \pm 0.1$ & $2.11 \pm 5.64$ \\
\hline MIDE & 1.1 & 40.4 & $0.0 \pm 0.1$ & $-1.85 \pm 8.70$ & 0.3 & $0.0 \pm 0.0$ & $2.21 \pm 5.75$ \\
\hline KAJ & 0.2 & 30.0 & $0.0 \pm 0.0$ & $-1.58 \pm 2.90$ & 0.3 & $0.0 \pm 0.0$ & $-0.26 \pm 2.33$ \\
\hline
\end{tabular}


Table S12. Biomass burning CO emissions and trends during 2010-2017 by region derived from Inversion \#3. Average biomass

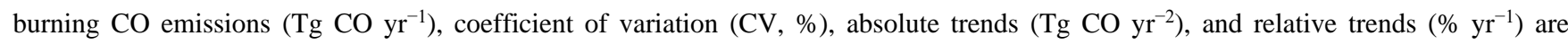
estimated for each region on the basis of Inversion \#3 and the prior GFED 4.1s inventory. Absolute and relative trends are both presented with $95 \%$ confidence limits. Significant trends are marked by asterisks $(* p<0.1, * * p<0.05$, and $* * * p<0.01)$.

\begin{tabular}{|c|c|c|c|c|c|c|c|}
\hline & $\begin{array}{c}\text { Average } \\
\left(\mathrm{Tg} \mathrm{CO} \mathrm{yr}^{-1}\right)\end{array}$ & $\begin{array}{l}\mathrm{CV} \\
(\%)\end{array}$ & $\begin{array}{c}\text { Absolute trend } \\
\text { (Inversion \#3) } \\
\left(\mathrm{Tg} \mathrm{CO} \mathrm{yr}^{-2}\right)\end{array}$ & $\begin{array}{c}\text { Relative trend } \\
\text { (Inversion \#3) } \\
\left(\% \mathrm{yr}^{-1}\right)\end{array}$ & $\begin{array}{c}\text { Average } \\
(\text { GFED 4.1s) } \\
\left(\mathrm{Tg} \mathrm{CO} \mathrm{yr}^{-1}\right)\end{array}$ & $\begin{array}{l}\text { Absolute trend } \\
\text { (GFED 4.1s) } \\
\left(\mathrm{Tg} \mathrm{CO} \mathrm{yr}^{-2} \text { ) }\right.\end{array}$ & $\begin{array}{c}\text { Relative trend } \\
\text { (GFED 4.1s) } \\
\left(\% \mathrm{yr}^{-1}\right)\end{array}$ \\
\hline EQAF & 83.9 & 9.5 & $1.7 \pm 3.0$ & $2.12 \pm 3.73$ & 69.1 & $0.2 \pm 1.7$ & $0.34 \pm 2.42$ \\
\hline SAF & 84.6 & 11.7 & $-3.0 \pm 3.1^{*}$ & $-2.86 \pm 2.94 *$ & 55.3 & $-1.2 \pm 0.8 * *$ & $-1.92 \pm 1.28 * *$ \\
\hline BRA & 55.3 & 37.2 & $1.3 \pm 8.9$ & $1.52 \pm 10.72$ & 33.4 & $-1.6 \pm 6.9$ & $-2.32 \pm 10.03$ \\
\hline SEAS & 43.5 & 69.7 & $0.4 \pm 13.2$ & $1.27 \pm 42.87$ & 46.1 & $2.3 \pm 15.4$ & $8.87 \pm 60.58$ \\
\hline RUS & 35.7 & 27.3 & $-0.6 \pm 4.2$ & $-1.58 \pm 11.77$ & 33.5 & $-0.4 \pm 5.0$ & $-1.69 \pm 19.04$ \\
\hline OCE & 26.1 & 48.9 & $-1.6 \pm 5.3$ & $-12.70 \pm 43.73$ & 14.0 & $-0.4 \pm 2.3$ & $-7.34 \pm 39.81$ \\
\hline NAF & 27.4 & 9.9 & $0.4 \pm 1.1$ & $1.64 \pm 4.93$ & 20.1 & $0.2 \pm 0.4$ & $1.17 \pm 2.02$ \\
\hline SSA & 17.6 & 55.2 & $-3.1 \pm 2.9^{* *}$ & $-7.75 \pm 7.42 * *$ & 10.4 & $-1.3 \pm 2.1$ & $-5.44 \pm 8.47$ \\
\hline CAN & 19.0 & 45.8 & $1.1 \pm 3.7$ & $8.57 \pm 29.41$ & 20.0 & $0.5 \pm 3.0$ & $2.90 \pm 16.45$ \\
\hline USA & 7.0 & 42.1 & $0.5 \pm 1.2$ & $9.98 \pm 23.95$ & 5.6 & $0.5 \pm 1.3$ & $11.90 \pm 31.11$ \\
\hline CAM & 4.5 & 58.0 & $-0.3 \pm 1.1$ & $-10.83 \pm 39.51$ & 4.6 & $0.0 \pm 0.7$ & $-1.01 \pm 21.42$ \\
\hline CAS & 4.1 & 48.5 & $0.0 \pm 0.9$ & $-0.48 \pm 14.44$ & 2.2 & $0.0 \pm 0.5$ & $1.12 \pm 19.70$ \\
\hline EU & 5.0 & 30.2 & $0.0 \pm 0.7$ & $1.24 \pm 17.63$ & 2.1 & $0.1 \pm 0.2$ & $6.00 \pm 12.26$ \\
\hline NSA & 2.2 & 65.4 & $0.2 \pm 0.6$ & $7.69 \pm 22.63$ & 3.3 & $0.1 \pm 0.4$ & $3.59 \pm 10.28$ \\
\hline $\mathrm{CHN}$ & 3.6 & 24.0 & $0.0 \pm 0.4$ & $0.22 \pm 11.14$ & 4.1 & $0.1 \pm 0.4$ & $2.69 \pm 10.37$ \\
\hline SAS & 2.6 & 26.3 & $0.0 \pm 0.3$ & $-1.41 \pm 10.00$ & 2.3 & $0.0 \pm 0.2$ & $1.05 \pm 9.00$ \\
\hline MIDE & 1.2 & 39.4 & $-0.1 \pm 0.2$ & $-4.40 \pm 9.27$ & 0.4 & $0.0 \pm 0.0$ & $-0.57 \pm 9.46$ \\
\hline KAJ & 0.2 & 26.9 & $0.0 \pm 0.0$ & $7.44 \pm 20.37$ & 0.3 & $0.0 \pm 0.0$ & $3.52 \pm 8.62$ \\
\hline
\end{tabular}




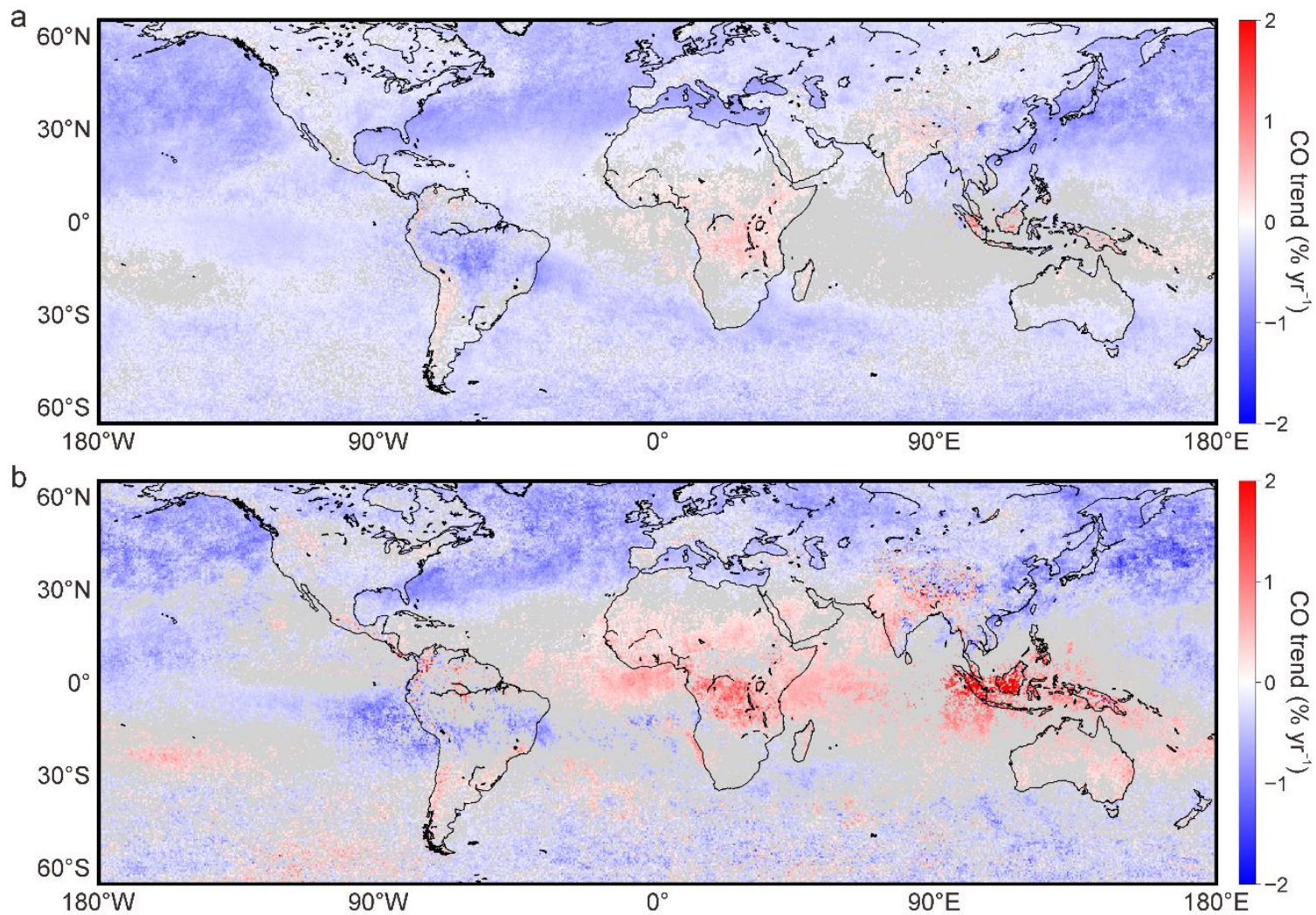

Figure S1. Trends in the MOPITT CO columns. The map (a) shows the 2005-2017 trends in MOPITT CO total columns at the spatial resolution of $0.5^{\circ} \times 0.5^{\circ}$, and the map (b) shows the trends from 2010 to 2017. The trends in (a) and (b) are both estimated on the base of monthly time series using a curve fitting method as described in Zheng et al. (2018). The grey colour in maps indicates the areas without 5 statistically significant trends $(p \geq 0.05)$. 


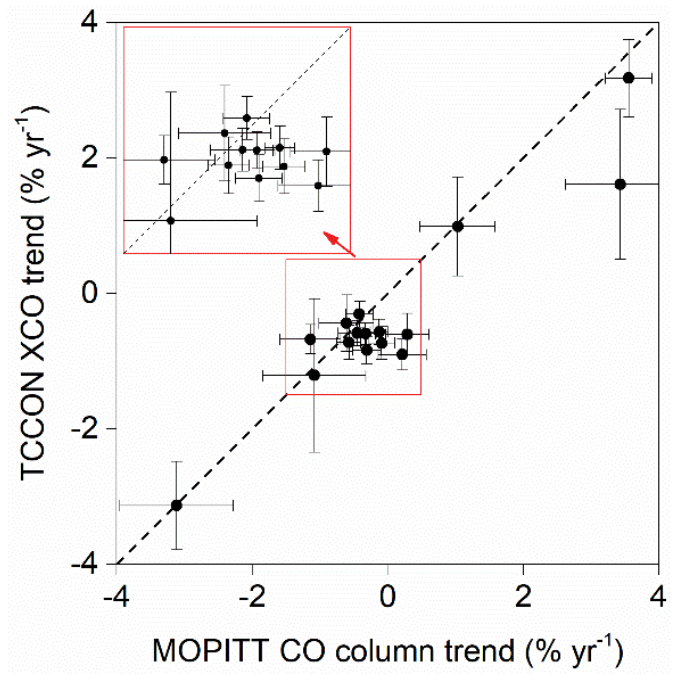

Figure S2. Comparison between the trends of MOPITT CO column and TCCON XCO. Each dot represents a site in TCCON archive plotted according to the observed XCO trend (y-axis) and the collocated CO column trend derived from MOPITT v7 retrievals ( $\mathrm{x}$-axis). These TCCON sites all present statistically significant trends $(p<0.05)$ with the error bars being 95\% confidence intervals. The MOPITT

$5 \mathrm{CO}$ columns are first averaged to generate monthly global maps at the spatial resolution of $0.5^{\circ} \times 0.5^{\circ}$, and then the grid cells that contain the TCCON sites are used in the comparison with TCCON observations. The trends shown in this figure are calculated based on monthly time series using a curve fitting method as described in Zheng et al. (2018). 

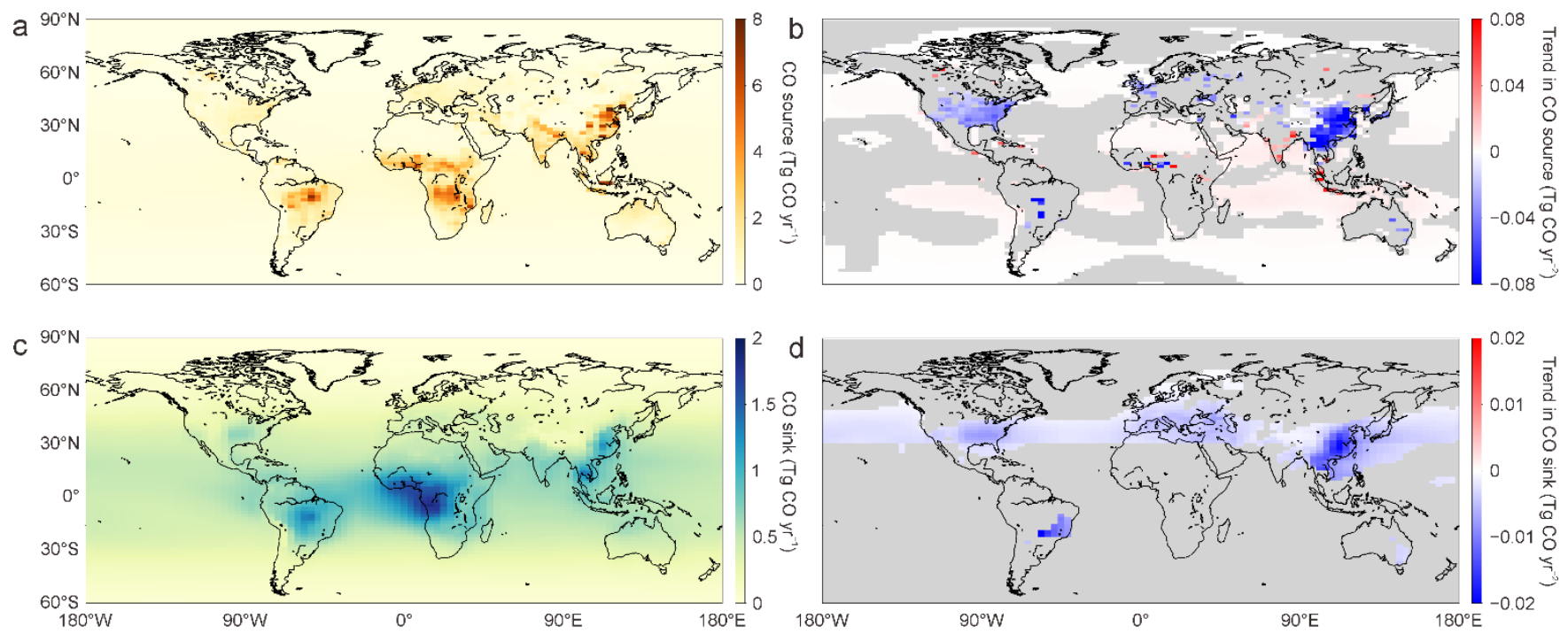

Figure S3. Spatial distribution of the global CO budget and 2005-2017 trends. Annual average CO total source and sink during $2005-$ 2017 are shown at the spatial resolution of $3.75^{\circ}$ longitude $\times 1.9^{\circ}$ latitude in (a) and (c), respectively, and linear trends of each grid cell are shown in (b) and (d), which are estimated using the linear least squares fitting method based on annual time series. Grey colour in (b) and

5 (d) indicates the areas without statistically significant trends $(p \geq 0.05)$. All data shown in this figure are derived from Inversion \#2 results. 

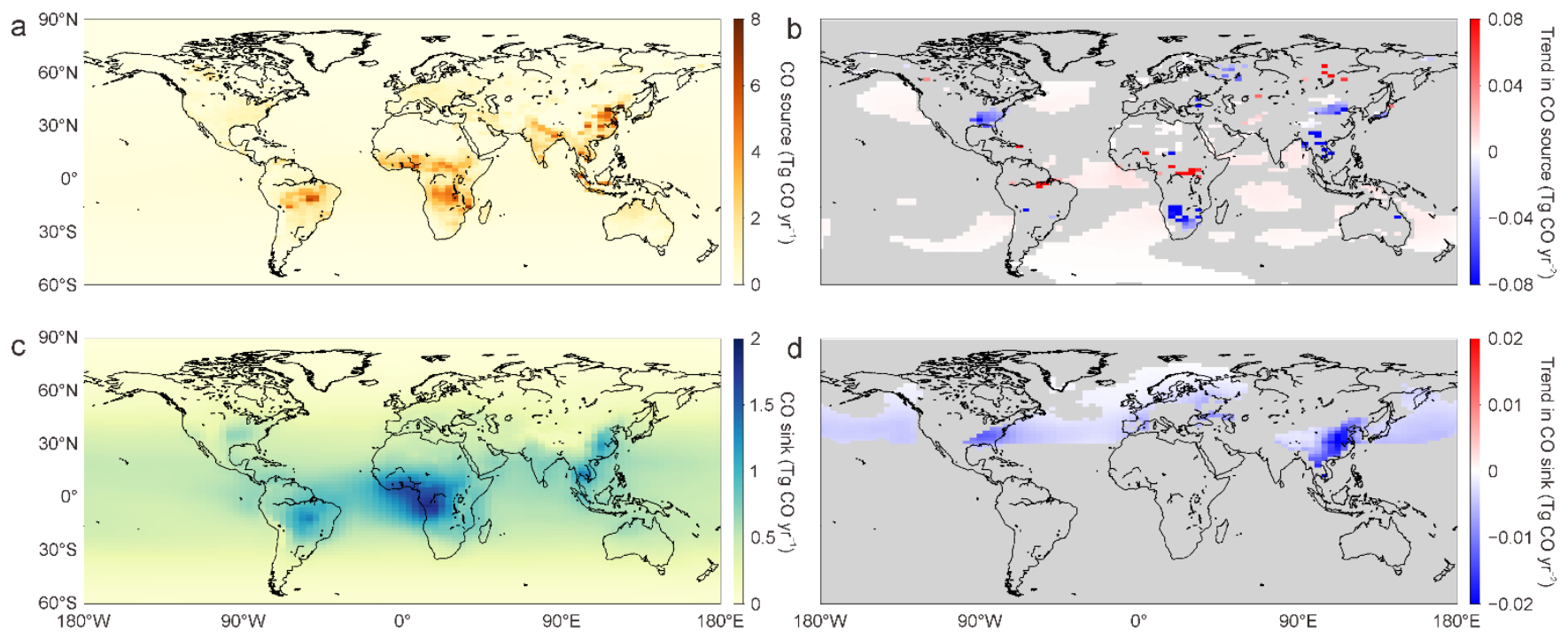

Figure S4. Spatial distribution of the global CO budget and 2010-2017 trends. Annual average CO total source and sink during 2010 2017 are shown at the spatial resolution of $3.75^{\circ}$ longitude $\times 1.9^{\circ}$ latitude in (a) and (c), respectively, and linear trends of each grid cell are shown in (b) and (d), which are estimated using the linear least squares fitting method based on annual time series. Grey colour in (b) and

5 (d) indicates the areas without statistically significant trends $(p \geq 0.05)$. All data shown in this figure are derived from Inversion \#3 results. 



Figure S5. Comparison of monthly biomass burning CO emissions between Inversion \#1 and GFED 4.1s. Average monthly biomass burning CO emissions are calculated on the basis of GFED 4.1s (black curve) and Inversion \#1 results (red curve) for EQAF (a), SAF (b), and BRA (c). The error bars represent one standard deviation of inter-annual variations between 2000 and 2017. 

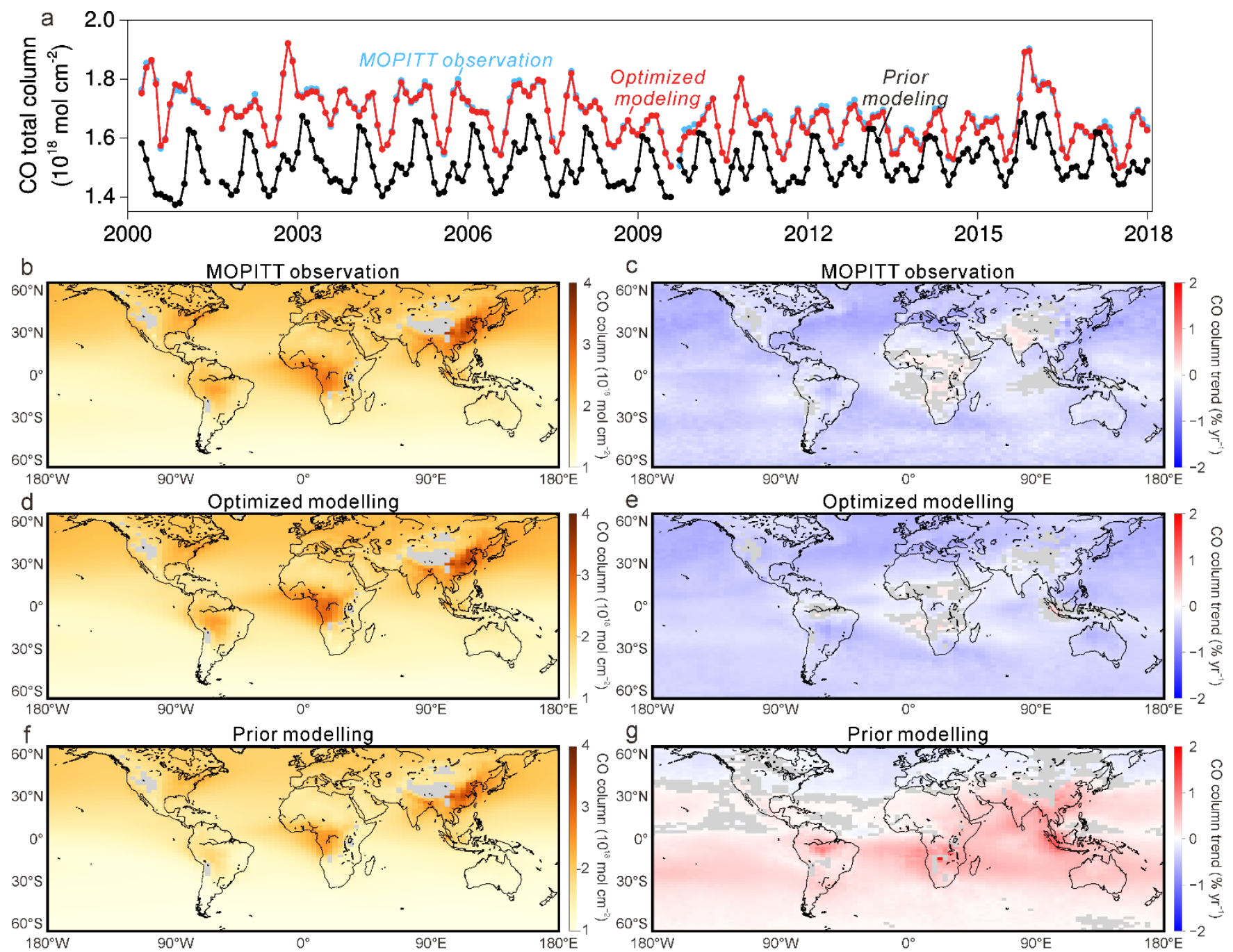

Figure S6. Evaluation of modelled CO total columns with MOPITT observations. Global monthly average CO total columns are presented in (a) with MOPITT observations (blue curve), modelling with prior emissions (black curve), and modelling with Inversion \#1 optimized emissions (red curve). Annual average CO total columns are presented at the spatial resolution of $3.75^{\circ}$ longitude $\times 1.9^{\circ}$ latitude

5 for MOPITT observations (b), Inversion \#1 modelling (d), and the prior modelling (f). Trends in CO total columns during 2000-2017 are presented at the spatial resolution of $3.75^{\circ}$ longitude $\times 1.9^{\circ}$ latitude for MOPITT observations (c), Inversion \#1 modelling (e), and the prior modelling (g). The trends in (c), (e), and (g) are estimated on the base of monthly time series using a curve fitting method as described in Zheng et al. (2018). Grey colour in all of the maps indicates the areas without valid data or statistically significant trends $(p \geq 0.05)$. 

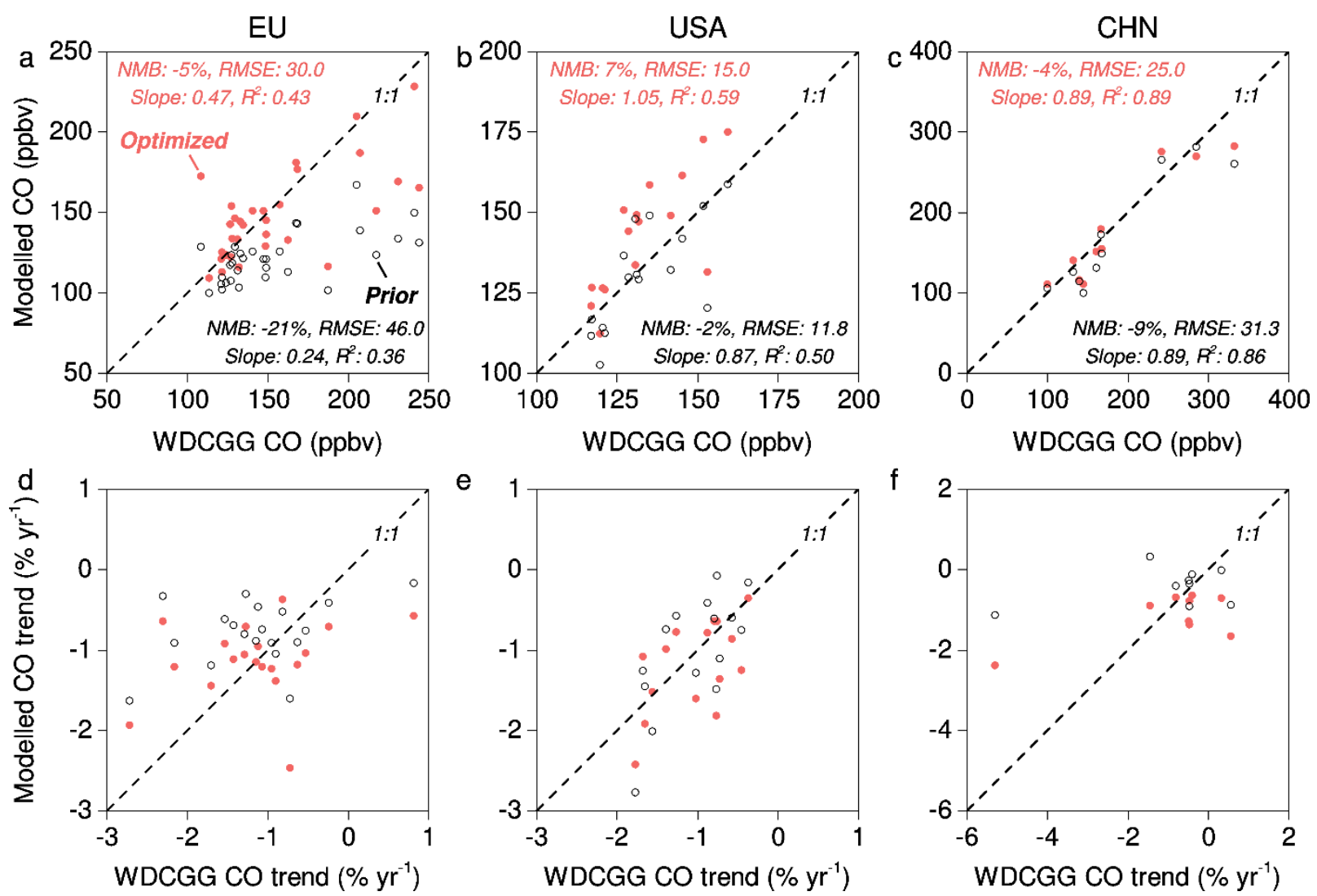

Figure S7. Evaluation of Inversion \#1 with the WDCGG observations by region. The comparison is conducted for annual average and 2000-2017 trends of surface CO concentrations between the modelled results and the WDCGG observations in EU (a, d), the USA (b, e), and CHN (c, f). The WDCGG sites used for each region are within the three black boxes shown in Fig. A1. The trends are calculated based

5 on monthly time series using a curve fitting method as described in Zheng et al. (2018). 

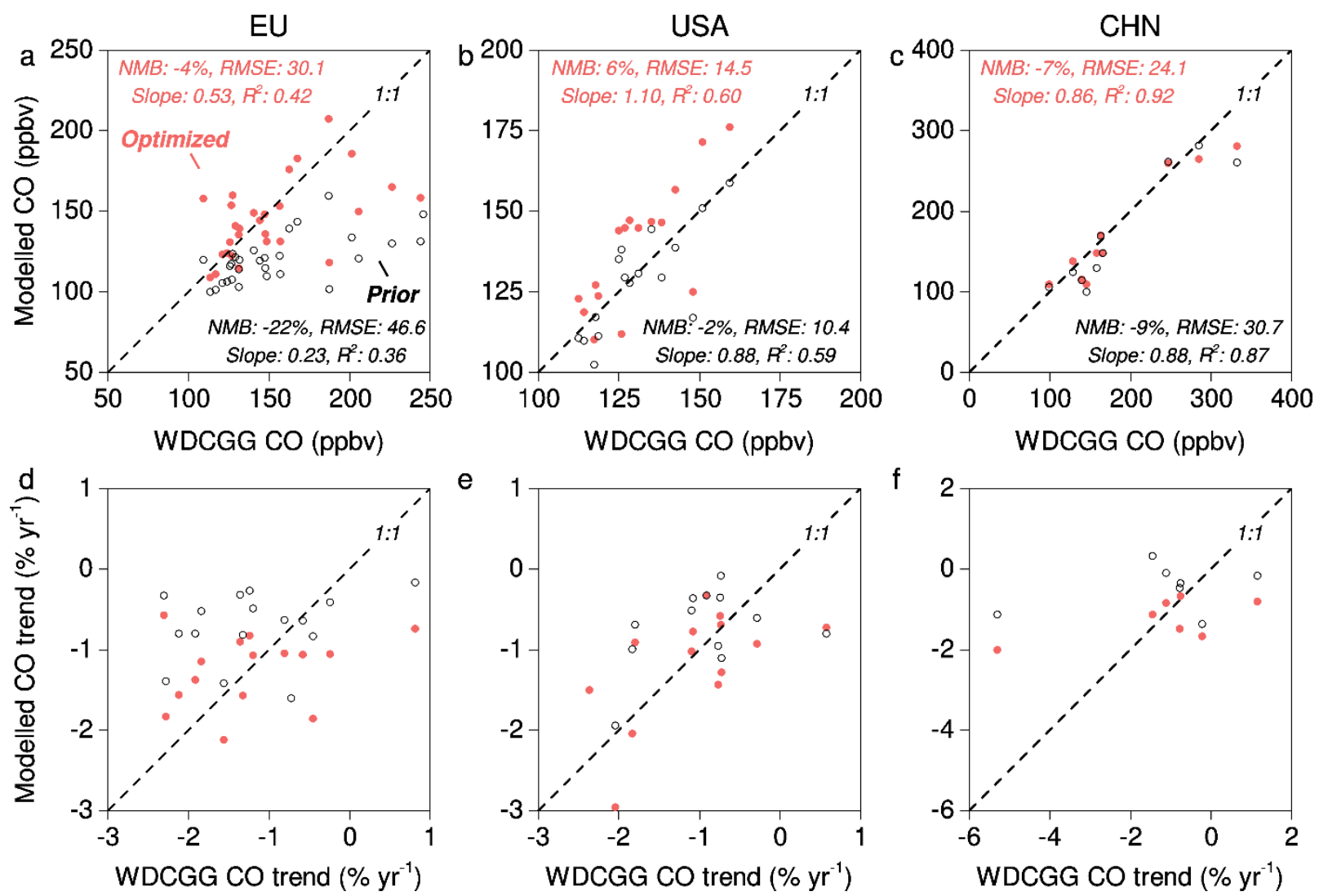

Figure S8. Evaluation of Inversion \#2 with the WDCGG observations by region. The comparison is conducted for annual average and 2005-2017 trends of surface CO concentrations between the modelled results and the WDCGG observations in EU (a, d), the USA (b, e), and CHN (c, f). The WDCGG sites used for each region are within the three black boxes shown in Fig. A1. The trends are calculated based

5 on monthly time series using a curve fitting method as described in Zheng et al. (2018). 



Figure S9. Evaluation of Inversion \#3 with the WDCGG observations by region. The comparison is conducted for annual average and 2010-2017 trends of surface CO concentrations between the modelled results and the WDCGG observations in EU (a, d), the USA (b, e), and CHN (c, f). The WDCGG sites used for each region are within the three black boxes shown in Fig. A1. The trends are calculated based

5 on monthly time series using a curve fitting method as described in Zheng et al. (2018). 


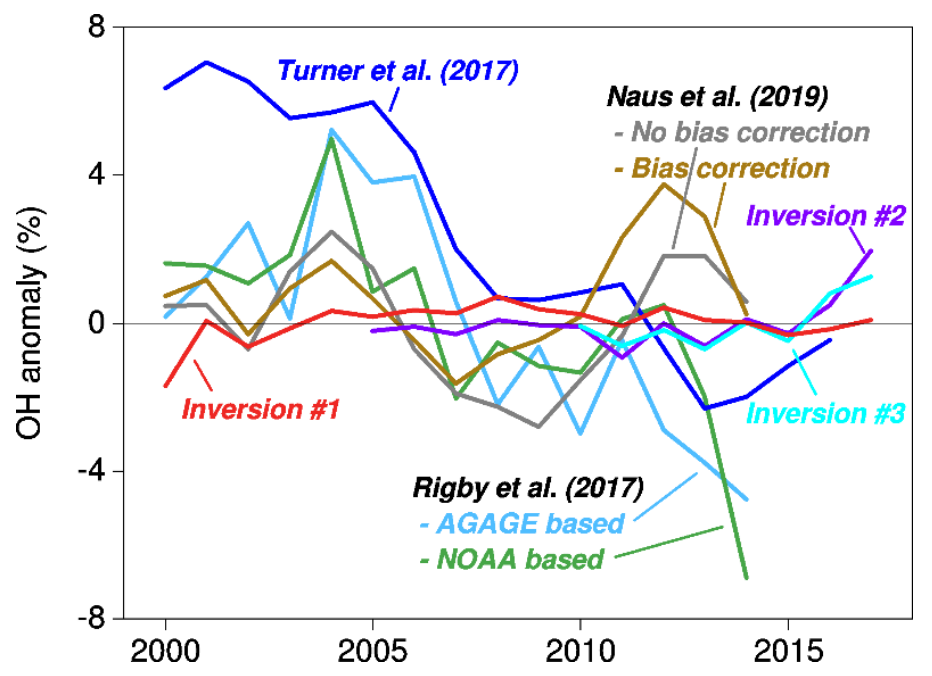

Figure S10. Comparison of $\mathrm{OH}$ anomalies between this study estimates and two-box model inversions from literature. This study estimates are derived from Inversion \#1 (red curve), Inversion \#2 (purple curve), and Inversion \#3 (cyan curve). Two-box model inversions are obtained from Turner et al. (2017) (dark blue curve), Rigby et al. (2017) (light blue and green curves), and Naus et al. 5 (2019) (grey and brown curves). 


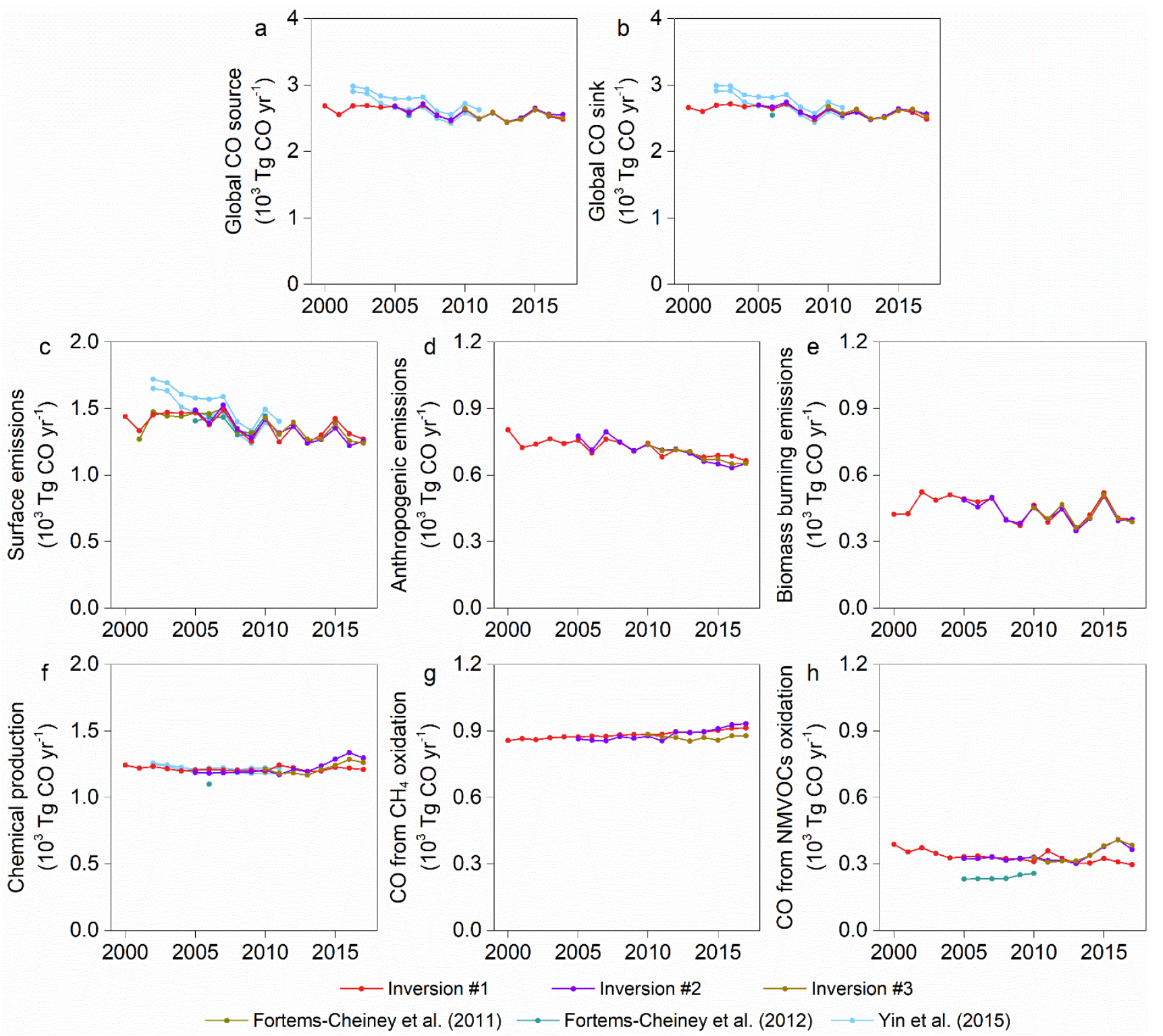

Figure S11. Comparison of Inversions \#1, \#2, and \#3 results with the estimates using previous versions of our inversion system. The comparison is conducted for the global CO source (a), the global CO sink (b), the surface direct emissions (c), the anthropogenic emissions (d), the biomass burning emissions (e), the $\mathrm{CO}$ chemical production (f), the $\mathrm{CO}$ production from $\mathrm{CH}_{4}$ oxidation (g), and the $\mathrm{CO}$ production 5 from NMVOCs oxidation (h). 


\section{References}

Arellano Jr., A. F., Kasibhatla, P. S., Giglio, L., van der Werf, G. R., and Randerson, J. T.: Top-down estimates of global CO sources using MOPITT measurements, Geophys. Res. Lett., 31, doi: 10.1029/2003GL018609, 2004.

Arellano Jr., A. F., Kasibhatla, P. S., Giglio, L., van der Werf, G. R., Randerson, J. T., and Collatz, G. J.: Time-dependent

5 inversion estimates of global biomass-burning CO emissions using Measurement of Pollution in the Troposphere (MOPITT) measurements, J. Geophys. Res. Atmos., 111, doi: 10.1029/2005JD006613, 2006.

Blumenstock, T., Hase, F., Schneider, M., García, O. E. and Sepúlveda, E.: TCCON data from Izana (ES), Release GGG2014.R1, doi:10.14291/tccon.ggg2014.izana01.r1, 2017.

De Mazière, M., Sha, M. K., Desmet, F., Hermans, C., Scolas, F., Kumps, N., Metzger, J.-M., Duflot, V. and Cammas, J.-P.:

10 TCCON data from Réunion Island (RE), Release GGG2014.R1, doi:10.14291/tccon.ggg2014.reunion01.r1, 2017.

Deutscher, N. M., Notholt, J., Messerschmidt, J., Weinzierl, C., Warneke, T., Petri, C. and Grupe, P.: TCCON data from Bialystok (PL), Release GGG2014.R1, doi:10.14291/tccon.ggg2014.bialystok01.r1/1183984, 2017.

Dubey, M. K., Henderson, B. G., Green, D., Butterfield, Z. T., Keppel-Aleks, G., Allen, N. T., Blavier, J.-F., Roehl, C. M., Wunch, D. and Lindenmaier, R.: TCCON data from Manaus (BR), Release GGG2014.R0,

15 doi:10.14291/tccon.ggg2014.manaus01.r0/1149274, 2017a.

Dubey, M. K., Lindenmaier, R., Henderson, B. G., Green, D., Allen, N. T., Roehl, C. M., Blavier, J.-F., Butterfield, Z. T., Love, S., Hamelmann, J. D. and Wunch, D.: TCCON data from Four Corners (US), Release GGG2014.R0, doi:10.14291/tccon.ggg2014.fourcorners01.r0/1149272, 2017b.

Feist, D. G., Arnold, S. G., John, N. and Geibel, M. C.: TCCON data from Ascension Island (SH), Release GGG2014.R0,

20 doi:10.14291/tccon.ggg2014.ascension01.r0/1149285, 2017.

Fortems-Cheiney, A., Chevallier, F., Pison, I., Bousquet, P., Szopa, S., Deeter, M. N., and Clerbaux, C.: Ten years of CO emissions as seen from Measurements of Pollution in the Troposphere (MOPITT), J. Geophys. Res. Atmos., 116, D05304, doi: 10.1029/2010JD014416, 2011.

Fortems-Cheiney, A., Chevallier, F., Pison, I., Bousquet, P., Saunois, M., Szopa, S., Cressot, C., Kurosu, T. P., Chance, K.,

25 and Fried, A.: The formaldehyde budget as seen by a global-scale multi-constraint and multi-species inversion system, Atmos. Chem. Phys., 12, 6699-6721, doi: 10.5194/acp-12-6699-2012, 2012.

Gaubert, B., Worden, H. M., Arellano, A. F. J., Emmons, L. K., Tilmes, S., Barré, J., Martinez Alonso, S., Vitt, F., Anderson, J. L., Alkemade, F., Houweling, S., and Edwards, D. P.: Chemical Feedback From Decreasing Carbon Monoxide Emissions, Geophys. Res. Lett., 44, 9985-9995, doi: 10.1002/2017GL074987, 2017.

30 Goo, T.-Y., Oh, Y.-S. and Velazco, V. A.: TCCON data from Anmeyondo (KR), Release GGG2014.R0, doi:10.14291/tccon.ggg2014.anmeyondo01.r0/1149284, 2017. 
Griffith, D. W. T., Velazco, V. A., Deutscher, N. M., Paton-Walsh, C., Jones, N. B., Wilson, S. R., Macatangay, R. C., Kettlewell, G. C., Buchholz, R. R. and Riggenbach, M. O.: TCCON data from Wollongong (AU), Release GGG2014.R0, doi:10.14291/tccon.ggg2014.wollongong01.r0/1149291, 2017a.

Griffith, D. W. T., Deutscher, N. M., Velazco, V. A., Wennberg, P. O., Yavin, Y., Keppel-Aleks, G., Washenfelder, R. A.,

5 Toon, G. C., Blavier, J.-F., Paton-Walsh, C., Jones, N. B., Kettlewell, G. C., Connor, B. J., Macatangay, R. C., Roehl, C., Ryczek, M., Glowacki, J., Culgan, T. and Bryant, G. W.: TCCON data from Darwin (AU), Release GGG2014.R0, doi:10.14291/tccon.ggg2014.darwin01.r0/1149290, 2017b.

Hase, F., Blumenstock, T., Dohe, S., Groß, J. and Kiel, M. ä.: TCCON data from Karlsruhe (DE), Release GGG2014.R1, doi:10.14291/tccon.ggg2014.karlsruhe01.r1/1182416, 2017.

10 Iraci, L. T., Podolske, J. R., Hillyard, P. W., Roehl, C., Wennberg, P. O., Blavier, J.-F., Landeros, J., Allen, N., Wunch, D., Zavaleta, J., Quigley, E., Osterman, G. B., Barrow, E. and Barney, J.: TCCON data from Indianapolis (US), Release GGG2014.R1, doi:10.14291/tccon.ggg2014.indianapolis01.r1/1330094, 2017a.

Iraci, L. T., Podolske, J. R., Hillyard, P. W., Roehl, C., Wennberg, P. O., Blavier, J.-F., Landeros, J., Allen, N., Wunch, D., Zavaleta, J., Quigley, E., Osterman, G. B., Albertson, R., Dunwoody, K. and Boyden, H.: TCCON data from Edwards (US),

15 Release GGG2014.R1, doi:10.14291/tccon.ggg2014.edwards01.r1/1255068, $2017 \mathrm{~b}$.

Jiang, Z., Worden, J. R., Worden, H., Deeter, M., Jones, D. B. A., Arellano, A. F., and Henze, D. K.: A 15-year record of CO emissions constrained by MOPITT CO observations, Atmos. Chem. Phys., 17, 4565-4583, doi: 10.5194/acp-17-4565-2017, 2017.

Kawakami, S., Ohyama, H., Arai, K., Okumura, H., Taura, C., Fukamachi, T. and Sakashita, M.: TCCON data from Saga (JP), Release GGG2014.R0, doi:10.14291/tccon.ggg2014.saga01.r0/1149283, 2017.

Kivi, R., Heikkinen, P. and Kyrö, E.: TCCON data from Sodankylä (FI), Release GGG2014.R0, doi:10.14291/tccon.ggg2014.sodankyla01.r0/1149280, 2017.

Kopacz, M., Jacob, D. J., Fisher, J. A., Logan, J. A., Zhang, L., Megretskaia, I. A., Yantosca, R. M., Singh, K., Henze, D. K., Burrows, J. P., Buchwitz, M., Khlystova, I., McMillan, W. W., Gille, J. C., Edwards, D. P., Eldering, A., Thouret, V., and

25 Nedelec, P.: Global estimates of $\mathrm{CO}$ sources with high resolution by adjoint inversion of multiple satellite datasets (MOPITT, AIRS, SCIAMACHY, TES), Atmos. Chem. Phys., 10, 855-876, doi: 10.5194/acp-10-855-2010, 2010.

Morino, I., Yokozeki, N., Matsuzaki, T. and Horikawa, M.: TCCON data from Rikubetsu (JP), Release GGG2014.R2, , doi:10.14291/tccon.ggg2014.rikubetsu01.r2, 2017a.

Morino, I., Matsuzaki, T. and Horikawa, M.: TCCON data from Tsukuba (JP), 125HR, Release GGG2014.R2, 30 doi:10.14291/tccon.ggg2014.tsukuba02.r2, 2017b.

Morino, I., Velazco, V. A., Hori, A., Uchino, O. and Griffith, D. W. T.: TCCON data from Burgos, Ilocos Norte (PH), Release GGG2014.R0, doi:10.14291/tccon.ggg2014.burgos01.r0, 2018. 
Müller, J.-F., Stavrakou, T., Bauwens, M., George, M., Hurtmans, D., Coheur, P.-F., Clerbaux, C., and Sweeney, C.: TopDown CO Emissions Based On IASI Observations and Hemispheric Constraints on OH Levels, Geophys. Res. Lett., 45, 1621-1629, doi:10.1002/2017GL076697, 2018.

Notholt, J., Warneke, T., Petri, C., Deutscher, N. M., Weinzierl, C., Palm, M. and Buschmann, M.: TCCON data from Ny

5 Ålesund, Spitsbergen (NO), Release GGG2014.R0, doi:10.14291/tccon.ggg2014.nyalesund01.r0/1149278, 2017a.

Notholt, J., Petri, C., Warneke, T., Deutscher, N. M., Palm, M., Buschmann, M., Weinzierl, C., Macatangay, R. C. and Grupe, P.: TCCON data from Bremen (DE), Release GGG2014.R0, doi:10.14291/tccon.ggg2014.bremen01.r0/1149275, $2017 b$.

Park, K., Emmons, L. K., Wang, Z., and Mak, J. E.: Joint Application of Concentration and $\delta^{18} \mathrm{O}$ to Investigate the Global 10 Atmospheric CO Budget, Atmosphere, 6, 547, 2015.

Pétron, G., Granier, C., Khattatov, B., Yudin, V., Lamarque, J.-F., Emmons, L., Gille, J., and Edwards, D. P.: Monthly CO surface sources inventory based on the 2000-2001 MOPITT satellite data, Geophys. Res. Lett., 31, n/a-n/a, doi: 10.1029/2004GL020560, 2004.

Rigby, M., Montzka, S. A., Prinn, R. G., White, J. W. C., Young, D., O’Doherty, S., Lunt, M. F., Ganesan, A. L., Manning,

15 A. J., Simmonds, P. G., Salameh, P. K., Harth, C. M., Mühle, J., Weiss, R. F., Fraser, P. J., Steele, L. P., Krummel, P. B., McCulloch, A., and Park, S.: Role of atmospheric oxidation in recent methane growth, Proc. Natl. Acad. Sci., 114, 5373, doi: 10.1073/pnas.1616426114, 2017.

Naus, S., Montzka, S. A., Pandey, S., Basu, S., Dlugokencky, E. J., and Krol, M.: Constraints and biases in a tropospheric two-box model of OH, Atmos. Chem. Phys., 19, 407-424, doi: 10.5194/acp-19-407-2019, 2019.

20 Sherlock, V., Connor, B., Robinson, J., Shiona, H., Smale, D. and Pollard, D. F.: TCCON data from Lauder (NZ), 120HR, Release GGG2014.R0, doi:10.14291/tccon.ggg2014.lauder01.r0/1149293, 2017a.

Sherlock, V., Connor, B., Robinson, J., Shiona, H., Smale, D. and Pollard, D. F.: TCCON data from Lauder (NZ), 125HR, Release GGG2014.R0, doi:10.14291/tccon.ggg2014.lauder02.r0/1149298, 2017b.

Stavrakou, T., and Müller, J.-F.: Grid-based versus big region approach for inverting CO emissions using Measurement of

25 Pollution in the Troposphere (MOPITT) data, J. Geophys. Res. Atmos., 111, doi: 10.1029/2005JD006896, 2006.

Strong, K., Roche, S., Franklin, J. E., Mendonca, J., Lutsch, E., Weaver, D., Fogal, P. F., Drummond, J. R., Batchelor, R. and Lindenmaier, R.: TCCON data from Eureka (CA), Release GGG2014.R3, doi:10.14291/tccon.ggg2014.eureka01.r3, 2018 .

Sussmann, R. and Rettinger, M.: TCCON data from Garmisch (DE), Release GGG2014.R2, 30 doi:10.14291/tccon.ggg2014.garmisch01.r2, 2017.

Sussmann, R. and Rettinger, M.: TCCON data from Zugspitze (DE), Release GGG2014.R1, doi:10.14291/tccon.ggg2014.zugspitze01.r1, 2018.

Té, Y., Jeseck, P. and Janssen, C.: TCCON data from Paris (FR), Release GGG2014.R0, doi:10.14291/tccon.ggg2014.paris01.r0/1149279, 2017. 
Turner, A. J., Frankenberg, C., Wennberg, P. O., and Jacob, D. J.: Ambiguity in the causes for decadal trends in atmospheric methane and hydroxyl, Proc. Natl. Acad. Sci., 114, 5367, doi: 10.1073/pnas.1616020114, 2017.

Warneke, T., Messerschmidt, J., Notholt, J., Weinzierl, C., Deutscher, N. M., Petri, C. and Grupe, P.: TCCON data from Orléans (FR), Release GGG2014.R0, doi:10.14291/tccon.ggg2014.orleans01.r0/1149276, 2017.

5 Wennberg, P. O., Wunch, D., Roehl, C. M., Blavier, J.-F., Toon, G. C. and Allen, N. T.: TCCON data from Caltech (US), Release GGG2014.R1, doi:10.14291/tccon.ggg2014.pasadena01.r1/1182415, 2017a.

Wennberg, P. O., Wunch, D., Yavin, Y., Toon, G. C., Blavier, J.-F., Allen, N. T. and Keppel-Aleks, G.: TCCON data from Jet Propulsion Laboratory (US), 2007, Release GGG2014.R0, doi:10.14291/tccon.ggg2014.jp101.r0/1149163, $2017 \mathrm{~b}$.

Wennberg, P. O., Roehl, C. M., Blavier, J.-F., Wunch, D. and Allen, N. T.: TCCON data from Jet Propulsion Laboratory 10 (US), 2011, Release GGG2014.R1, doi:10.14291/tccon.ggg2014.jp102.r1/1330096, 2017c.

Wennberg, P. O., Roehl, C. M., Wunch, D., Toon, G. C., Blavier, J.-F., Washenfelder, R., Keppel-Aleks, G., Allen, N. T. and Ayers, J.: TCCON data from Park Falls (US), Release GGG2014.R1, doi:10.14291/tccon.ggg2014.parkfalls01.r1, 2017d.

Wennberg, P. O., Wunch, D., Roehl, C. M., Blavier, J.-F., Toon, G. C. and Allen, N. T.: TCCON data from Lamont (US),

15 Release GGG2014.R1, doi:10.14291/tccon.ggg2014.lamont01.r1/1255070, 2017e.

Wunch, D., Mendonca, J., Colebatch, O., Allen, N. T., Blavier, J.-F., Roche, S., Hedelius, J., Neufeld, G., Springett, S., Worthy, D., Kessler, R. and Strong, K.: TCCON data from East Trout Lake, SK (CA), Release GGG2014.R1, doi:10.14291/tccon.ggg2014.easttroutlake01.r1, 2017.

Yin, Y., Chevallier, F., Ciais, P., Broquet, G., Fortems-Cheiney, A., Pison, I., and Saunois, M.: Decadal trends in global CO

20 emissions as seen by MOPITT, Atmos. Chem. Phys., 15, 13433-13451, doi: 10.5194/acp-15-13433-2015, 2015.

Yin, Y., Ciais, P., Chevallier, F., van der Werf, G. R., Fanin, T., Broquet, G., Boesch, H., Cozic, A., Hauglustaine, D., Szopa, S., and Wang, Y.: Variability of fire carbon emissions in equatorial Asia and its nonlinear sensitivity to El Niño, Geophys. Res. Lett., 43, 10,472-410,479, doi: 10.1002/2016GL070971, 2016.

Zheng, B., Chevallier, F., Ciais, P., Yin, Y., Deeter, M., Worden, H., Wang, Y. L., Zhang, Q., and He, K. B.: Rapid decline

25 in carbon monoxide emissions and export from East Asia between years 2005 and 2016, Environ. Res. Lett., 13, 044007, doi: 10.1088/1748-9326/aab2b3, 2018. 